\title{
EXISTENCE, UNIQUENESS AND APPROXIMATION OF A DOUBLY-DEGENERATE NONLINEAR PARABOLIC SYSTEM MODELLING BACTERIAL EVOLUTION
}

\author{
JOHN W. BARRETT \\ Department of Mathematics, \\ Imperial College, London SW7 2AZ, UK. \\ jwb@ic.ac.uk \\ KLAUS DECKELNICK \\ Institut für Analysis and Numerik, \\ Otto-von-Guericke-Universität Magdeburg, \\ Universitätsplatz 2, D-39106 Magdeburg, Germany. \\ Klaus.Deckelnick@mathematik.uni-magdeburg.de
}

We consider the following nonlinear parabolic system

$$
\begin{aligned}
& \frac{\partial u}{\partial t}-c \Delta u=-f(u) v \quad \text { in } \Omega_{T}:=\Omega \times(0, T), \quad \Omega \subset \mathbb{R}^{d}, \\
& \frac{\partial v}{\partial t}-\nabla \cdot(b(u) \nabla[\psi(v)])=\theta f(u) v \quad \text { in } \Omega_{T}
\end{aligned}
$$

subject to no flux boundary conditions, and non-negative initial data $u^{0}$ and $v^{0}$ on $u$ and $v$. Here we assume that $c>0, \theta \geq 0$ and that $f \in C_{\mathrm{loc}}^{0,1}([0, \infty))$ is increasing with $f(0)=0$. The system is possibly doubly-degenerate in that $b \in C_{\text {loc }}^{1,1}([0, \infty))$ is only nonnegative, and $\psi \in C^{1}([0, \infty)) \cap C^{2}((0, \infty))$ is convex, strictly increasing with $\psi(0)=0$ and possibly $\psi^{\prime}(0)=0$. The above models the spatiotemporal evolution of a bacterium species on a thin film of nutrient, where $u$ is the nutrient concentration and $v$ is the bacterial cell density. Under some further mild technical assumptions on $b$ and $\psi$, we prove the existence and uniqueness of a weak solution to the above system. Moreover, we prove error bounds for a fully practical finite element approximation of this system. All of our results apply to the choices $b(r):=r^{q}$ and $\psi(r):=r^{p}$ with $q \geq 2$ and $p \geq 1$, for example.

Keywords: Doubly-degenerate parabolic system, porous medium equation, bacterial pattern formation, existence, uniqueness, finite elements, error analysis.

AMS Subject Classification: 35K65, 35K55, 65M15, 65M60 


\section{Introduction}

In this paper we consider the initial boundary value problem:

(P) Find functions $u, v: \Omega_{T}:=\Omega \times(0, T) \rightarrow \mathbb{R}_{\geq 0}$ such that

$$
\begin{aligned}
& \frac{\partial u}{\partial t}-c \Delta u=-f(u) v \quad \text { in } \Omega_{T}, \\
& \frac{\partial v}{\partial t}-\nabla \cdot(b(u) \nabla[\psi(v)])=\theta f(u) v \quad \text { in } \Omega_{T}, \\
& \frac{\partial u}{\partial \nu}=b(u) \frac{\partial[\psi(v)]}{\partial \nu}=0 \quad \text { on } \partial \Omega \times(0, T), \\
& u(\cdot, 0)=u^{0}(\cdot), \quad v(\cdot, 0)=v^{0}(\cdot) \quad \text { in } \Omega ;
\end{aligned}
$$

where $\Omega$ is a bounded domain in $\mathbb{R}^{d}$, with a $C^{2}$ boundary $\partial \Omega$ if $d \geq 2, \nu$ is normal to $\partial \Omega$ and $T>0$ is a fixed positive time. In the above the constants $c$ and $\theta$ are positive and non-negative, respectively. We start by formulating our assumptions on the data. Let $b \in C_{\text {loc }}^{1,1}([0, \infty))$ with $b(r) \geq 0$ for $r \geq 0$. We assume, in addition, that for every $R>0$ there exists a $C_{R} \geq 0$ such that

$$
\left[b^{\prime}(r)\right]^{2} \leq C_{R} b(r) \quad \text { for all } r \in[0, R] .
$$

Furthermore, we assume that

$$
f \in C_{\mathrm{loc}}^{0,1}([0, \infty)) \text { is increasing with } f(0)=0 ;
$$

and that $\psi \in C^{1}([0, \infty)) \cap C^{2}((0, \infty))$ is convex, strictly increasing with

$$
\psi(0)=0 \quad \text { and } \quad \limsup _{s \rightarrow 0} \frac{s \psi^{\prime \prime}(s)}{\psi^{\prime}(s)}<\infty .
$$

We note, for example, that $b(r):=r^{q}$ and $\psi(s):=s^{p}$, with $q \geq 2$ and $p \geq 1$, satisfy our assumptions; whereas, for example, $\psi(s):=\int_{0}^{s} \exp \left(-\left(r^{-p}\right)\right) d r$, with $p>0$, does not satisfy the second condition in (1.4). Concerning the initial data, we assume that

$$
\begin{aligned}
& \quad u^{0} \in W^{2-\frac{2}{p^{*}}, p^{*}}(\Omega) \cap H^{2}(\Omega) \quad \text { for } p^{*}>\max \{d, 2\}, \quad v^{0} \in H^{1}(\Omega) \\
& \text { with } \quad 0 \leq u^{0} \leq \bar{u}, \quad 0 \leq v^{0} \leq \bar{v} \quad \text { in } \Omega, \quad \frac{\partial u^{0}}{\partial \nu}=0 \quad \text { on } \partial \Omega
\end{aligned}
$$

where $\bar{u}, \bar{v} \in \mathbb{R}_{\geq 0}$.

A system of the type (P) was proposed in Kawasaki et al. ${ }^{5}$ to model the spatiotemporal evolution of a bacterium species on a thin film of nutrient, where $u$ is the nutrient concentration and $v$ is the bacterial cell density. The diffusion coefficients of the nutrient and bacterial cells are $c>0$ and $b(u) \psi^{\prime}(v) \geq 0$, respectively. Thus under our assumptions on $b$ and $\psi$ above, this bacterial coefficient is possibly doubly-degenerate. The term $f(u) v \geq 0$ represents the consumption rate of nutrient by the cells; while $\theta f(u) v$ is the growth rate of bacteria, where $\theta \geq 0$ is the conversion rate of consumed nutrient to bacterial growth. For example, the choice $f(r):=\frac{\rho r}{1+\gamma r}$, with $\rho, \gamma>0$, gives rise to Michaelis-Menten kinetics. 
In fact, the Kawasaki et al. ${ }^{5}$ model is precisely the system (1.1a)-(1.1d) with $b(r):=r, \psi(s):=\frac{1}{2} s^{2}$ and the above Michaelis-Menten $f(r)$. These choices of $f$ and $\psi$ satisfy our general assumptions (1.3) and (1.4), respectively. However, this choice of $b$ does not satisfy our assumption (1.2). As stated in Kawasaki et al. ${ }^{5}$ the degeneracy of the bacterial coefficient $b(u) \psi^{\prime}(v)$ has not been empirically determined, so they opt for the simplest choice $b(r):=r$ and $\psi^{\prime}(s):=s$. However, from a modelling point of view the choice $b(r):=r^{q}$ with $q \geq 2$ is just as good; and this satisfies (1.2).

This Kawasaki et al. ${ }^{5}$ model was studied in Barrett and Nürnberg ${ }^{1}$ with $d \leq 2$, and for a more general $f$ : increasing $f \in C_{\mathrm{loc}}^{0,1}([0, \infty))$ satisfying $f(0)=0$. There a fully practical finite element approximation was introduced and analysed. Stability results, leading to subsequence convergence, and hence an existence proof for this model was established. In addition, numerical experiments were presented. However, no uniqueness proof, and hence no error analysis for the numerical approximation, was presented in Barrett and Nürnberg. ${ }^{1}$ It is our crucial assumption on $b,(1.2)$, that enables us to establish the uniqueness proof for $(\mathrm{P})$ here, and the subsequent error analysis for its finite element approximation. However, we do not expect the qualitative features of the Kawasaki et al. ${ }^{5}$ model to change with the introduction of this assumption.

The possible double degeneracy of the $v$ diffusion coefficient makes the analysis of this system particularly difficult. In fact we are unaware of any existence proof or any numerical analysis work on such a system, apart from that in Barrett and Nürnberg ${ }^{1}$; even though there is a great deal of interest in the degenerate system $(\mathrm{P})$ in the mathematical/theoretical biology community, see e.g. Golding et al., ${ }^{4}$ Mimura et al. ${ }^{7}$ and the references therein. Related models describing bacterial pattern formation are also discussed in these review papers.

The outline of this paper is as follows. In the next section, we establish existence of a solution to $(\mathrm{P})$; via a regularised problem $\left(\mathrm{P}_{\varepsilon, \delta}\right)$, where $\varepsilon>0$ and $\delta>0$ are the regularization parameters for $\psi$ and $b$ respectively. We establish existence of a solution to $\left(\mathrm{P}_{\varepsilon, \delta}\right)$, and various $\varepsilon$ and $\delta$ independent bounds on its solution. This enables us to pass to the limits, first as $\delta \searrow 0$ then as $\varepsilon \searrow 0$, to establish the existence of solutions to $\left(\mathrm{P}_{\varepsilon}\right)$ and $(\mathrm{P})$, respectively. Moreover, the assumption (1.2) on $b$ enables us to establish the uniqueness of these solutions to $\left(\mathrm{P}_{\varepsilon}\right)$ and $(\mathrm{P})$. At the end of Section 2, we establish an error bound between the unique solutions of $\left(\mathrm{P}_{\varepsilon}\right)$ and $(\mathrm{P})$. In Section 3 we introduce a fully practical finite element approximation of $\left(\mathrm{P}_{\varepsilon}\right)$, and hence of $(\mathrm{P})$. Moreover, we establish existence, uniqueness, stability bounds and an error estimate for this approximation. Here we extend the approach in Nochetto and Verdi ${ }^{8}$ for degenerate parabolic problems, such as the porous medium equation, to the possibly doubly-degenerate parabolic system $(\mathrm{P})$.

Finally, we end this section with a few comments about notation. We adopt the standard notation for Sobolev spaces, denoting the norm of $W^{m, q}(G)\left(m \in \mathbb{N}, q \in[1, \infty]\right.$ and $G$ a bounded domain in $\mathbb{R}^{d}$ with a Lipschitz 
boundary) by $\|\cdot\|_{m, q, G}$ and the semi-norm by $|\cdot|_{m, q, G}$. For $q=2, W^{m, 2}(G)$ will be denoted by $H^{m}(G)$ with the associated norm and semi-norm written, as respectively, $\|\cdot\|_{m, G}$ and $|\cdot|_{m, G}$. For ease of notation, in the common case when $G \equiv \Omega$ the subscript " $\Omega$ " will be dropped on the above norms and semi-norms. We introduce also $\left(H^{1}(\Omega)\right)^{\prime}$, as the dual space of $H^{1}(\Omega)$, and denote its norm by $\|\cdot\|_{\left(H^{1}\right)^{\prime}}$. These are naturally extended to vector and matrix functions. Throughout $(\cdot, \cdot)$ denotes the standard $L^{2}$ inner product over $\Omega$, and $\langle\cdot, \cdot\rangle$ denotes the duality pairing between $\left(H^{1}(\Omega)\right)^{\prime}$ and $H^{1}(\Omega)$. In addition, $C$ denotes a generic constant independent of the regularization parameters $\varepsilon, \delta$ and the mesh parameters $h, \tau$. Finally, $C_{\gamma_{1}, \cdots, \gamma_{\ell}}$ denotes a constant dependent on $\left\{\gamma_{i}^{-1}\right\}_{i=1}^{\ell}$.

\section{Existence and Uniqueness}

\subsection{The Regularized Problem}

The function $b$ is degenerate if $b(r)=0$ for some $r \in[0, \bar{u}]$, whereas $\psi$ is degenerate if $\psi^{\prime}(0)=0$. In order to prove existence for $(\mathrm{P})$ we first regularise $b$, and regularise $\psi$ if it is degenerate. For $\delta>0$, we set

$$
b_{\delta}(r):=b(r)+\delta \quad \forall r \geq 0 .
$$

If $\psi^{\prime}(0)=0$, then for $\varepsilon>0$ let $q_{\varepsilon}:=\inf \left\{s \geq 0: \psi^{\prime}(s)=\varepsilon\right\}$ so that $q_{\varepsilon} \rightarrow 0$ as $\varepsilon \rightarrow 0$; otherwise, we set $q_{\varepsilon}=0$. We then define

$$
\psi_{\varepsilon}(s):= \begin{cases}\varepsilon s & s \in\left[0, q_{\varepsilon}\right] \\ \psi(s)+\varepsilon q_{\varepsilon}-\psi\left(q_{\varepsilon}\right) & s \geq q_{\varepsilon} .\end{cases}
$$

Clearly, $\psi_{\varepsilon} \in C^{1}\left(\mathbb{R}_{\geq 0}\right)$; and for $\varepsilon$ sufficiently small, $\varepsilon \in\left(0, \varepsilon_{0}\right]$, we have that

$$
\psi(s) \leq \psi_{\varepsilon}(s) \leq \psi(s)+\varepsilon q_{\varepsilon} \quad \text { and } \quad \psi_{\varepsilon}^{\prime}(s) \geq \varepsilon \quad \forall s \geq 0 .
$$

We now consider the following regularised system for a given $\varepsilon \in\left(0, \varepsilon_{0}\right]$ and $\delta>0$ :

$\left(\mathbf{P}_{\varepsilon, \delta}\right)$ Find functions $u_{\varepsilon, \delta}, v_{\varepsilon, \delta}: \Omega_{T} \rightarrow \mathbb{R}_{\geq 0}$ such that

$$
\begin{aligned}
& \frac{\partial u_{\varepsilon, \delta}}{\partial t}-c \Delta u_{\varepsilon, \delta}=-f\left(u_{\varepsilon, \delta}\right) v_{\varepsilon, \delta} \quad \text { in } \Omega_{T}, \\
& \frac{\partial v_{\varepsilon, \delta}}{\partial t}-\nabla \cdot\left(b_{\delta}\left(u_{\varepsilon, \delta}\right) \nabla\left[\psi_{\varepsilon}\left(v_{\varepsilon, \delta}\right)\right]\right)=\theta f\left(u_{\varepsilon, \delta}\right) v_{\varepsilon, \delta} \quad \text { in } \Omega_{T} \text {, } \\
& \frac{\partial u_{\varepsilon, \delta}}{\partial \nu}=\frac{\partial v_{\varepsilon, \delta}}{\partial \nu}=0 \quad \text { on } \partial \Omega \times(0, T), \\
& u_{\varepsilon, \delta}(\cdot, 0)=u^{0}(\cdot), \quad v_{\varepsilon, \delta}(\cdot, 0)=\max \left\{v^{0}(\cdot), \delta\right\} \quad \text { in } \Omega .
\end{aligned}
$$

Theorem 2.1. Under our stated assumptions on the data and coefficients in Section 1, it follows for given $\varepsilon \in\left(0, \varepsilon_{0}\right]$ and $\delta>0$ that there exists a unique solution 
$\left(u_{\varepsilon, \delta}, v_{\varepsilon, \delta}\right)$ to $\left(P_{\varepsilon, \delta}\right)$ such that

$$
\begin{aligned}
& u_{\varepsilon, \delta} \in L^{p^{*}}\left(0, T ; W^{2, p^{*}}(\Omega)\right) \cap L^{p^{*}}\left(0, T ; W^{1, \infty}(\Omega)\right), \quad \frac{\partial u_{\varepsilon, \delta}}{\partial t} \in L^{p^{*}}\left(\Omega_{T}\right), \\
& v_{\varepsilon, \delta} \in L^{\infty}\left(0, T ; H^{1}(\Omega)\right), \quad \psi_{\varepsilon}\left(v_{\varepsilon, \delta}\right) \in L^{2}\left(0, T ; H^{2}(\Omega)\right), \quad \frac{\partial v_{\varepsilon, \delta}}{\partial t} \in L^{2}\left(\Omega_{T}\right), \\
& 0 \leq u_{\varepsilon, \delta} \leq \bar{u}, \quad \delta \leq v_{\varepsilon, \delta} \leq v^{*}:=\bar{v} \exp (\theta f(\bar{u}) T) \quad \text { in } \Omega_{T} ;
\end{aligned}
$$

where $p^{*}$ is as in (1.5).

Proof. Our strategy to prove existence to $\left(\mathrm{P}_{\varepsilon, \delta}\right)$ consists in first proving existence of a solution to a suitably modified problem, and then to show in a second step that we have actually obtained a solution of the original system. In order to formulate and solve our auxiliary problem we truncate the nonlinearities $f, b$ and $\psi$, and hence $b_{\delta}$ and $\psi_{\varepsilon}$. First, we choose $\bar{f} \in C^{0}(\mathbb{R})$ and $\bar{b} \in C^{1}(\mathbb{R})$ such that

$$
\begin{aligned}
& 0 \leq \bar{f}(r) \leq \bar{C}, \quad 0 \leq \bar{b}(r) \leq \bar{C}, \quad\left|\bar{b}^{\prime}(r)\right| \leq \bar{C} \quad \forall r \in \mathbb{R} ; \\
& \bar{f}(r)=0 \quad \forall r \leq 0 ; \quad \bar{f}(r)=f(r), \quad \bar{b}(r)=b(r) \quad \forall r \in[0, \bar{u}] .
\end{aligned}
$$

Next for $s \geq 0$ let

$$
\bar{\psi}(s):= \begin{cases}\psi(s) & s \in\left[0, v^{*}\right] \\ \psi\left(v^{*}\right)+\left(s-v^{*}\right) \psi^{\prime}\left(v^{*}\right) & s>v^{*}\end{cases}
$$

We then define $\bar{b}_{\delta}$ and $\bar{\psi}_{\varepsilon}$ as in (2.1) and (2.2) with $b$ and $\psi$ replaced by $\bar{b}$ and $\bar{\psi}$, respectively. We then set $\bar{\psi}(s):=-\bar{\psi}(-s)$ and $\bar{\psi}_{\varepsilon}(s):=-\bar{\psi}_{\varepsilon}(-s)$ for $s \leq 0$; and so $\bar{b}_{\delta} \in C^{1}(\mathbb{R})$ and $\bar{\psi}, \bar{\psi}_{\varepsilon} \in C^{1}(\mathbb{R})$ with $\delta \leq \bar{b}_{\delta}(s), \varepsilon \leq \bar{\psi}_{\varepsilon}^{\prime}(s) \leq \psi^{\prime}\left(v^{*}\right)$ for all $s \in \mathbb{R}$. Finally, let $\bar{g} \in C(\mathbb{R})$ be such that

$$
\bar{g}(s):=\min \left(|s|, v^{*}\right) \quad \forall s \in \mathbb{R} .
$$

Consider the following auxiliary problem: Find functions $u_{\varepsilon, \delta}, w_{\varepsilon, \delta}: \Omega_{T} \rightarrow \mathbb{R}$ such that

$$
\begin{aligned}
\frac{\partial u_{\varepsilon, \delta}}{\partial t}-c \Delta u_{\varepsilon, \delta} & =-\bar{f}\left(u_{\varepsilon, \delta}\right) \bar{g}\left(v_{\varepsilon, \delta}\right) & & \text { in } \Omega_{T}, \\
\frac{\partial w_{\varepsilon, \delta}}{\partial t}-\alpha^{\varepsilon} \Delta w_{\varepsilon, \delta}-\gamma^{\varepsilon} \cdot \nabla w_{\varepsilon, \delta} & =\theta \bar{\psi}_{\varepsilon}^{\prime}\left(v_{\varepsilon, \delta}\right) \bar{f}\left(u_{\varepsilon, \delta}\right) \bar{g}\left(v_{\varepsilon, \delta}\right) & & \text { in } \Omega_{T}, \\
\frac{\partial u_{\varepsilon, \delta}}{\partial \nu}=\frac{\partial w_{\varepsilon, \delta}}{\partial \nu} & =0 & & \text { on } \partial \Omega \\
u_{\varepsilon, \delta}(\cdot, 0)=u^{0}(\cdot), \quad w_{\varepsilon, \delta}(\cdot, 0) & =\bar{\psi}_{\varepsilon}\left(\max \left\{v^{0}(\cdot), \delta\right\}\right) & & \text { in } \Omega
\end{aligned}
$$

where $v_{\varepsilon, \delta}=\left(\bar{\psi}_{\varepsilon}\right)^{-1}\left(w_{\varepsilon, \delta}\right), \alpha^{\varepsilon}=\bar{\psi}_{\varepsilon}^{\prime}\left(v_{\varepsilon, \delta}\right) \bar{b}_{\delta}\left(u_{\varepsilon, \delta}\right)$ and $\gamma^{\varepsilon}=\bar{\psi}_{\varepsilon}^{\prime}\left(v_{\varepsilon, \delta}\right) \bar{b}_{\delta}^{\prime}\left(u_{\varepsilon, \delta}\right) \nabla u_{\varepsilon, \delta}$.

Let $X:=L^{2}\left(\Omega_{T}\right)$ and define the operator $S: X \times X \rightarrow X \times X$ as follows: given $(u, w) \in X \times X$, set $v:=\left(\bar{\psi}_{\varepsilon}\right)^{-1}(w)$ and denote by $\tilde{u} \in L^{\infty}\left(0, T ; H^{1}(\Omega)\right) \cap$ 
$L^{2}\left(0, T ; H^{2}(\Omega)\right)$ the unique solution of the inhomogeneous heat equation

$$
\begin{aligned}
\frac{\partial \tilde{u}}{\partial t}-c \Delta \tilde{u} & =-\bar{f}(u) \bar{g}(v) & & \text { in } \Omega_{T}, \\
\frac{\partial \tilde{u}}{\partial \nu} & =0 & & \text { on } \partial \Omega \times(0, T), \\
\tilde{u}(\cdot, 0) & =u^{0}(\cdot) & & \text { in } \Omega .
\end{aligned}
$$

Linear parabolic $L^{p}$ theory implies, see Solonnikov, ${ }^{11}$ on recalling our assumptions on $u^{0}$ and $\partial \Omega$ that

$$
\begin{aligned}
\sup _{t \in(0, T)}\|\tilde{u}(\cdot, t)\|_{1, p^{*}}^{p^{*}} & +\int_{0}^{T}\left(\left\|\frac{\partial \tilde{u}}{\partial t}\right\|_{0, p^{*}}^{p^{*}}+\|\tilde{u}\|_{2, p^{*}}^{p^{*}}\right) d t \\
& \leq C\left(\left\|u^{0}\right\|_{2-\frac{2}{p^{*}}, p^{*}}^{p^{*}}+\int_{0}^{T}\|\bar{f}(u) \bar{g}(v)\|_{0, p^{*}}^{p^{*}} d t\right) \leq C
\end{aligned}
$$

uniformly in $(u, w)$. As $p^{*}>d$, the embedding $W^{1, p^{*}}(\Omega) \hookrightarrow C(\bar{\Omega})$ is continuous and we obtain that

$$
\int_{0}^{T}\|\tilde{u}\|_{1, \infty}^{p^{*}} d t \leq C
$$

uniformly in $(u, w)$.

Next, let $\tilde{w} \in L^{\infty}\left(0, T ; H^{1}(\Omega)\right) \cap L^{2}\left(0, T ; H^{2}(\Omega)\right)$ be the unique solution of the linear convection-diffusion equation

$$
\begin{aligned}
\frac{\partial \tilde{w}}{\partial t}-\alpha \Delta \tilde{w}-\gamma \cdot \nabla \tilde{w} & =\theta \bar{\psi}_{\varepsilon}^{\prime}(v) \bar{f}(u) \bar{g}(v) & & \text { in } \Omega_{T} \\
\frac{\partial \tilde{w}}{\partial \nu} & =0 & & \text { on } \partial \Omega \times(0, T), \\
\tilde{w}(\cdot, 0) & =\psi_{\varepsilon}\left(\max \left\{v^{0}(\cdot), \delta\right\}\right) & & \text { in } \Omega ;
\end{aligned}
$$

where $\alpha=\bar{\psi}_{\varepsilon}^{\prime}(v) \bar{b}_{\delta}(u)$ and $\gamma=\bar{\psi}_{\varepsilon}^{\prime}(v) \bar{b}_{\delta}^{\prime}(u) \nabla \tilde{u}$. Note that $\alpha \in L^{\infty}\left(\Omega_{T}\right)$ with $\alpha \geq$ $\varepsilon \delta$ in $\Omega_{T}$. The existence of $\tilde{w}$ can be proved via a suitable regularization of $\alpha$; e.g. extend $\alpha$ from $\Omega$ to $\mathbb{R}^{d}$ by $\varepsilon \delta$ and mollify, see e.g. p197 in Ladyzhenskaya et al. ${ }^{6}$ The crucial a priori bound is obtained by multiplying (2.11a) by $-\Delta \tilde{w}$. In view of the properties of $\bar{b}_{\delta}, \bar{\psi}_{\varepsilon}, \bar{f}$ and $\bar{g}$, one obtains that

$$
\begin{aligned}
\frac{1}{2} \frac{d}{d t}\|\nabla \tilde{w}\|_{0}^{2}+\varepsilon \delta\|\Delta \tilde{w}\|_{0}^{2} & \leq C\left[1+\|\nabla \tilde{u}\|_{0, \infty}\|\nabla \tilde{w}\|_{0}\right]\|\Delta \tilde{w}\|_{0} \\
& \leq \frac{\varepsilon \delta}{2}\|\Delta \tilde{w}\|_{0}^{2}+C_{\varepsilon, \delta}\left[1+\|\nabla \tilde{u}\|_{0, \infty}^{2}\|\nabla \tilde{w}\|_{0}^{2}\right] .
\end{aligned}
$$

A Gronwall argument, (2.10), elliptic regularity theory and (2.11a) imply that

$$
\sup _{t \in(0, T)}\|\nabla \tilde{w}(\cdot, t)\|_{0}^{2}+\int_{0}^{T}\left(\left\|\frac{\partial \tilde{w}}{\partial t}\right\|_{0}^{2}+\|\tilde{w}\|_{2}^{2}\right) d t \leq C_{\varepsilon, \delta}
$$

uniformly in $(u, w)$. 
We then set $S(u, w):=(\tilde{u}, \tilde{w})$. Note that the bounds $(2.9),(2.12)$ together with a well-known compactness result (see e.g. Simon ${ }^{10}$ ) show that $S(X \times X)$ is precompact in $X \times X$.

Let us now prove that $S$ is continuous. Suppose that $\left(u_{n}, w_{n}\right)_{n \in \mathbb{N}}$ converges to $(u, w)$ in $X \times X$. Let $\left(\tilde{u}_{n}, \tilde{w}_{n}\right)=S\left(u_{n}, w_{n}\right)$ and $(\tilde{u}, \tilde{w})=S(u, w)$. After passing to a subsequence, we may assume that

$$
u_{n} \rightarrow u, \quad w_{n} \rightarrow w, \quad v_{n}:=\left(\bar{\psi}_{\varepsilon}\right)^{-1}\left(w_{n}\right) \rightarrow\left(\bar{\psi}_{\varepsilon}\right)^{-1}(w)=: v \quad \text { a.e. in } \Omega_{T} .
$$

As a consequence, $\bar{f}\left(u_{n}\right) \bar{g}\left(v_{n}\right) \rightarrow \bar{f}(u) \bar{g}(v)$ a.e. in $\Omega_{T}$ and in $L^{p^{*}}\left(\Omega_{T}\right)$. Applying (2.9) to the difference $\tilde{u}_{n}-\tilde{u}$ and recalling the continuous embedding $W^{1, p^{*}}(\Omega) \hookrightarrow$ $C(\bar{\Omega})$ we deduce that

$$
\begin{aligned}
\int_{0}^{T}\left\|\tilde{u}_{n}-\tilde{u}\right\|_{1, \infty}^{2} d t & \leq C\left(\int_{0}^{T}\left\|\tilde{u}_{n}-\tilde{u}\right\|_{2, p^{*}}^{p^{*}} d t\right)^{\frac{2}{p^{*}}} \\
& \leq C\left(\int_{0}^{T}\left\|\bar{f}\left(u_{n}\right) \bar{g}\left(v_{n}\right)-\bar{f}(u) \bar{g}(v)\right\|_{0, p^{*}}^{p^{*}} d t\right)^{\frac{2}{p^{*}}} \\
& \rightarrow 0 \quad \text { as } n \rightarrow \infty
\end{aligned}
$$

Let us next write

$$
\begin{aligned}
\frac{\partial\left(\tilde{w}_{n}-\tilde{w}\right)}{\partial t}-\alpha_{n} \Delta\left(\tilde{w}_{n}-\tilde{w}\right)=\left(\alpha_{n}-\alpha\right) \Delta \tilde{w}+\gamma_{n} \cdot \nabla \tilde{w}_{n}-\gamma \cdot \nabla \tilde{w} \\
+\theta \bar{\psi}_{\varepsilon}^{\prime}\left(v_{n}\right) \bar{f}\left(u_{n}\right) \bar{g}\left(v_{n}\right)-\theta \bar{\psi}_{\varepsilon}^{\prime}(v) \bar{f}(u) \bar{g}(v)
\end{aligned}
$$

where $\alpha=\bar{\psi}_{\varepsilon}^{\prime}(v) \bar{b}_{\delta}(u), \alpha_{n}=\bar{\psi}_{\varepsilon}^{\prime}\left(v_{n}\right) \bar{b}_{\delta}\left(u_{n}\right), \gamma=\bar{\psi}_{\varepsilon}^{\prime}(v) \bar{b}_{\delta}^{\prime}(u) \nabla \tilde{u}$ and $\gamma_{n}=$ $\bar{\psi}_{\varepsilon}^{\prime}\left(v_{n}\right) \bar{b}_{\delta}^{\prime}\left(u_{n}\right) \nabla \tilde{u}_{n}$. Arguing in a similar way as in deriving (2.12) and using (2.13), as well as Lebesgue's convergence theorem, we obtain for a subsequence that

$$
\int_{0}^{T}\left(\left\|\frac{\partial\left(\tilde{w}_{n}-\tilde{w}\right)}{\partial t}\right\|_{0}^{2}+\left\|\tilde{w}_{n}-\tilde{w}\right\|_{2}^{2}\right) d t \rightarrow 0 \quad \text { as } \quad n \rightarrow \infty .
$$

In particular we have that $S\left(u_{n}, w_{n}\right) \rightarrow S(u, w)$ in $X \times X$ for a subsequence, from which we deduce that $S$ is continuous.

Schauder's fixed point theorem now implies that $S$ has a fixed point $\left(u_{\varepsilon, \delta}, w_{\varepsilon, \delta}\right) \in$ $X \times X$. Clearly, we have that $u_{\varepsilon, \delta}, w_{\varepsilon, \delta} \in L^{\infty}\left(0, T ; H^{1}(\Omega)\right) \cap L^{2}\left(0, T ; H^{2}(\Omega)\right)$ with $\frac{\partial u_{\varepsilon, \delta}}{\partial t}, \frac{\partial w_{\varepsilon, \delta}}{\partial t} \in L^{2}\left(\Omega_{T}\right)$ and $\left(u_{\varepsilon, \delta}, w_{\varepsilon, \delta}\right)$ is a solution of $(2.8 \mathrm{a})-(2.8 \mathrm{~d})$. If we let $v_{\varepsilon, \delta}:=$ $\left(\bar{\psi}_{\varepsilon}\right)^{-1}\left(w_{\varepsilon, \delta}\right)$, we find that $\frac{\partial w_{\varepsilon, \delta}}{\partial t}=\bar{\psi}_{\varepsilon}^{\prime}\left(v_{\varepsilon, \delta}\right) \frac{\partial v_{\varepsilon, \delta}}{\partial t}$ and $(2.8 \mathrm{~b})$ can be rewritten as

$$
\frac{\partial v_{\varepsilon, \delta}}{\partial t}-\nabla \cdot\left(\bar{b}_{\delta}\left(u_{\varepsilon, \delta}\right) \nabla\left[\bar{\psi}_{\varepsilon}\left(v_{\varepsilon, \delta}\right)\right]\right)=\theta \bar{f}\left(u_{\varepsilon, \delta}\right) \bar{g}\left(v_{\varepsilon, \delta}\right)
$$

It remains to show that $\left(u_{\varepsilon, \delta}, v_{\varepsilon, \delta}\right)$ is a solution of $\left(\mathrm{P}_{\varepsilon, \delta}\right),(2.4 \mathrm{a})-(2.4 \mathrm{~d})$. Multiplying $(2.8 \mathrm{a})$ by $\left(u_{\varepsilon, \delta}\right)^{-}:=\min \left\{u_{\varepsilon, \delta}, 0\right\}$, we deduce that

$$
\frac{1}{2} \frac{d}{d t}\left\|\left(u_{\varepsilon, \delta}\right)^{-}\right\|_{0}^{2}+c\left\|\nabla\left(u_{\varepsilon, \delta}\right)^{-}\right\|_{0}^{2}=-\int_{\left\{u_{\varepsilon, \delta} \leq 0\right\}} \bar{f}\left(u_{\varepsilon, \delta}\right) \bar{g}\left(v_{\varepsilon, \delta}\right) u_{\varepsilon, \delta}=0
$$

in view of (2.6) and (2.7). On recalling (1.5), the above yields that $u_{\varepsilon, \delta} \geq 0$ in $\Omega_{T}$. If we apply a similar argument with $\left(u_{\varepsilon, \delta}-\bar{u}\right)^{+}$and take into account that 
$\bar{f}\left(u_{\varepsilon, \delta}\right) \bar{g}\left(v_{\varepsilon, \delta}\right) \geq 0$ and (1.5), we obtain that $u_{\varepsilon, \delta} \leq \bar{u}$ in $\Omega_{T}$; and hence that $\bar{f}\left(u_{\varepsilon, \delta}\right)=f\left(u_{\varepsilon, \delta}\right)$, as well as $\bar{b}\left(u_{\varepsilon, \delta}\right)=b\left(u_{\varepsilon, \delta}\right)$ and $\bar{b}_{\delta}\left(u_{\varepsilon, \delta}\right)=b_{\delta}\left(u_{\varepsilon, \delta}\right)$. Next, multiplying (2.14) by $\left(v_{\varepsilon, \delta}-\delta\right)^{-}$; since $\bar{f}\left(u_{\varepsilon, \delta}\right) \bar{g}\left(v_{\varepsilon, \delta}\right) \geq 0$, we infer that $v_{\varepsilon, \delta} \geq \delta$ in $\Omega_{T}$. In order to obtain an upper bound on $v_{\varepsilon, \delta}$, let $z(t):=\bar{v} \exp (\theta f(\bar{u}) t) \leq v^{*}$. Since $f$ is increasing and $u_{\varepsilon, \delta} \in[0, \bar{u}]$, we have that $f\left(u_{\varepsilon, \delta}\right) \leq f(\bar{u})$. Furthermore, on recalling (2.7), $\bar{g}\left(v_{\varepsilon, \delta}\right) \in\left[\delta, v_{\varepsilon, \delta}\right]$ as $v_{\varepsilon, \delta} \geq \delta$ in $\Omega_{T}$. Hence it follows that

$$
\begin{aligned}
\frac{\partial\left(v_{\varepsilon, \delta}-z\right)}{\partial t}-\nabla \cdot\left(\bar{b}_{\delta}\left(u_{\varepsilon, \delta}\right) \bar{\psi}_{\varepsilon}^{\prime}\left(v_{\varepsilon, \delta}\right) \nabla\left(v_{\varepsilon, \delta}-z\right)\right) & =\theta\left[f\left(u_{\varepsilon, \delta}\right) \bar{g}\left(v_{\varepsilon, \delta}\right)-f(\bar{u}) z\right] \\
& \leq \theta f(\bar{u})\left(v_{\varepsilon, \delta}-z\right) .
\end{aligned}
$$

Multiplying the above by $\left(v_{\varepsilon, \delta}-z\right)^{+}$, and recalling that $v^{0} \leq \bar{v}$, we deduce that $v_{\varepsilon, \delta} \leq z$ in $\Omega_{T}$. In conclusion, we have that $\left(u_{\varepsilon, \delta}, v_{\varepsilon, \delta}\right)$ satisfy the bounds (2.5c); so that $\bar{g}\left(v_{\varepsilon, \delta}\right)=v_{\varepsilon, \delta}$ and $\bar{\psi}_{\varepsilon}\left(v_{\varepsilon, \delta}\right)=\psi_{\varepsilon}\left(v_{\varepsilon, \delta}\right)$. Therefore it follows that $\left(u_{\varepsilon, \delta}, v_{\varepsilon, \delta}\right)$ is a solution of $\left(\mathrm{P}_{\varepsilon, \delta}\right),(2.4 \mathrm{a})-(2.4 \mathrm{~d})$, satisfying $(2.5 \mathrm{a})-(2.5 \mathrm{c})$.

Finally, it is easy to establish that this solution is unique. For example, one can simply follow the arguments used in the more subtle proof of uniqueness for the solution of the doubly-degenerate limit problem $(\mathrm{P})$ given in Theorem 2.3 below; on noting that $\psi_{\varepsilon}$ is also convex.

Corollary 2.1. Let the assumptions of Theorem 2.1 hold. Then there exists an $\varepsilon_{1} \in\left(0, \varepsilon_{0}\right]$, such that for $\varepsilon \in\left(0, \varepsilon_{1}\right]$ and $\delta>0$ we have the following bounds on $\left(u_{\varepsilon, \delta}, v_{\varepsilon, \delta}, w_{\varepsilon, \delta}:=\psi_{\varepsilon}\left(v_{\varepsilon, \delta}\right)\right)$ :

$$
\begin{aligned}
& \sup _{t \in(0, T)}\left\|u_{\varepsilon, \delta}(\cdot, t)\right\|_{1, p^{*}}^{p^{*}}+\int_{0}^{T}\left(\left\|\frac{\partial u_{\varepsilon, \delta}}{\partial t}\right\|_{1}^{2}+\left\|u_{\varepsilon, \delta}\right\|_{2, p^{*}}^{p^{*}}+\left\|u_{\varepsilon, \delta}\right\|_{1, \infty}^{p^{*}}\right) d t \leq C, \\
& \sup _{t \in(0, T)}\left\|w_{\varepsilon, \delta}(\cdot, t)\right\|_{1}^{2}+\int_{0}^{T}\left(\int_{\Omega} b_{\delta}\left(u_{\varepsilon, \delta}\right) \psi_{\varepsilon}^{\prime}\left(v_{\varepsilon, \delta}\right)\left|\Delta w_{\varepsilon, \delta}\right|^{2}\right) d t \leq C, \\
& \sup _{t \in(0, T)}\left\|\frac{\partial v_{\varepsilon, \delta}}{\partial t}(\cdot, t)\right\|_{\left(H^{1}\right)^{\prime}}^{2} \leq C \\
& \int_{0}^{T}\left\|\frac{\partial w_{\varepsilon, \delta}}{\partial t}\right\|_{0}^{2} d t \leq C \int_{0}^{T}\left\|\left[\psi_{\varepsilon}^{\prime}\left(v_{\varepsilon, \delta}\right)\right]^{\frac{1}{2}} \frac{\partial v_{\varepsilon, \delta}}{\partial t}\right\|_{0}^{2} d t \leq C .
\end{aligned}
$$

Proof. The inequality (2.15a), apart from the bound on $\int_{0}^{T}\left|\frac{\partial u_{\varepsilon, \delta}}{\partial t}\right|_{1}^{2} d t$, follows from (2.5c), (2.9) and (2.10). Next, multiplying $(2.4 \mathrm{~b})$ by $-\psi_{\varepsilon}^{\prime}\left(v_{\varepsilon, \delta}\right) \Delta w_{\varepsilon, \delta}$, on recalling $(2.5 \mathrm{~b})$, we obtain that

$$
\begin{aligned}
-\left(\frac{\partial w_{\varepsilon, \delta}}{\partial t}, \Delta w_{\varepsilon, \delta}\right)+ & \left(b_{\delta}\left(u_{\varepsilon, \delta}\right) \psi_{\varepsilon}^{\prime}\left(v_{\varepsilon, \delta}\right) \Delta w_{\varepsilon, \delta}, \Delta w_{\varepsilon, \delta}\right) \\
& =-\left(b_{\delta}^{\prime}\left(u_{\varepsilon, \delta}\right) \nabla u_{\varepsilon, \delta} \cdot \nabla w_{\varepsilon, \delta}+\theta f\left(u_{\varepsilon, \delta}\right) v_{\varepsilon, \delta}, \psi_{\varepsilon}^{\prime}\left(v_{\varepsilon, \delta}\right) \Delta w_{\varepsilon, \delta}\right) .
\end{aligned}
$$

Using integration by parts, and noting that $\nabla\left[f\left(u_{\varepsilon, \delta}\right)\right]=f^{\prime}\left(u_{\varepsilon, \delta}\right) \nabla u_{\varepsilon, \delta}$ a.e. in $\Omega_{T}$, 
see e.g. Theorem 2.1.11 in Ziemer ${ }^{12}$; we may infer that

$$
\begin{aligned}
\frac{1}{2} \frac{d}{d t}\left|w_{\varepsilon, \delta}\right|_{1}^{2}+ & \left(b_{\delta}\left(u_{\varepsilon, \delta}\right) \psi_{\varepsilon}^{\prime}\left(v_{\varepsilon, \delta}\right) \Delta w_{\varepsilon, \delta}, \Delta w_{\varepsilon, \delta}\right) \\
=-\left(b_{\delta}^{\prime}\left(u_{\varepsilon, \delta}\right) \nabla u_{\varepsilon, \delta} \cdot \nabla w_{\varepsilon, \delta}, \psi_{\varepsilon}^{\prime}\left(v_{\varepsilon, \delta}\right) \Delta w_{\varepsilon, \delta}\right) & \\
& +\theta\left(f^{\prime}\left(u_{\varepsilon, \delta}\right) v_{\varepsilon, \delta} \nabla u_{\varepsilon, \delta}, \psi_{\varepsilon}^{\prime}\left(v_{\varepsilon, \delta}\right) \nabla w_{\varepsilon, \delta}\right) \\
& +\theta\left(f\left(u_{\varepsilon, \delta}\right) \nabla w_{\varepsilon, \delta}, \nabla w_{\varepsilon, \delta}\right)+\theta\left(f\left(u_{\varepsilon, \delta}\right) v_{\varepsilon, \delta} \psi_{\varepsilon}^{\prime \prime}\left(v_{\varepsilon, \delta}\right) \nabla v_{\varepsilon, \delta}, \nabla w_{\varepsilon, \delta}\right) \\
=: & I_{1}+I_{2}+I_{3}+I_{4} .
\end{aligned}
$$

Recalling (1.2) and (2.5c) we have that

$$
\left|I_{1}\right| \leq \frac{1}{2}\left(b_{\delta}\left(u_{\varepsilon, \delta}\right) \psi_{\varepsilon}^{\prime}\left(v_{\varepsilon, \delta}\right) \Delta w_{\varepsilon, \delta}, \Delta w_{\varepsilon, \delta}\right)+C\left|u_{\varepsilon, \delta}\right|_{1, \infty}^{2}\left|w_{\varepsilon, \delta}\right|_{1}^{2} .
$$

Next, (2.5c) and (2.15a) imply that

$$
\left|I_{2}\right|+\left|I_{3}\right| \leq C\left(1+\left|w_{\varepsilon, \delta}\right|_{1}^{2}\right)
$$

In order to analyse $I_{4}$, we first note that (1.4) yields the existence of $\rho>0$ and $C_{0}>0$ such that

$$
s \psi^{\prime \prime}(s) \leq C_{0} \psi^{\prime}(s) \quad \text { for } s \in(0, \rho) \text {. }
$$

We may assume that $\rho>q_{\varepsilon}$ for $\varepsilon \in\left(0, \varepsilon_{1}\right]$, where $\varepsilon_{1}$ is sufficiently small. Since $\psi^{\prime}(s) \geq \psi^{\prime}(\rho)>0$ for $s \geq \rho$ we deduce that

$$
s \psi_{\varepsilon}^{\prime \prime}(s)=s \psi^{\prime \prime}(s) \leq K \psi^{\prime}(s)=K \psi_{\varepsilon}^{\prime}(s) \quad \text { for } s \in\left[\max \left\{q_{\varepsilon}, \delta\right\}, v^{*}\right],
$$

where $K=\max \left\{C_{0}, \frac{1}{\psi^{\prime}(\rho)} v^{*} \max _{s \in\left[\rho, v^{*}\right]} \psi^{\prime \prime}(s)\right\}$. This inequality also trivially holds for $s \in\left[\delta, q_{\varepsilon}\right]$. Thus we obtain from $(2.16)$ and $(2.5 \mathrm{c})$ that

$$
\left|I_{4}\right| \leq C \int_{\Omega} v_{\varepsilon, \delta} \psi_{\varepsilon}^{\prime \prime}\left(v_{\varepsilon, \delta}\right)\left|\nabla v_{\varepsilon, \delta}\right|\left|\nabla w_{\varepsilon, \delta}\right| \leq C \int_{\Omega} \psi_{\varepsilon}^{\prime}\left(v_{\varepsilon, \delta}\right)\left|\nabla v_{\varepsilon, \delta}\right|\left|\nabla w_{\varepsilon, \delta}\right|=C\left|w_{\varepsilon, \delta}\right|_{1}^{2} .
$$

Collecting the above bounds together yields that

$$
\frac{1}{2} \frac{d}{d t}\left|w_{\varepsilon, \delta}\right|_{1}^{2}+\frac{1}{2}\left(b_{\delta}\left(u_{\varepsilon, \delta}\right) \psi_{\varepsilon}^{\prime}\left(v_{\varepsilon, \delta}\right) \Delta w_{\varepsilon, \delta}, \Delta w_{\varepsilon, \delta}\right) \leq C+C\left(1+\left|u_{\varepsilon, \delta}\right|_{1, \infty}^{2}\right)\left|w_{\varepsilon, \delta}\right|_{1}^{2}
$$

and so a Gronwall argument together with (2.15a) and (2.5c) then implies (2.15b).

The bound (2.15c) follows from $(2.4 \mathrm{~b}),(2.5 \mathrm{c}),(2.15 \mathrm{~b})$. The bound $(2.15 \mathrm{~d})$ follows from multiplying $(2.4 \mathrm{~b})$ by $\psi_{\varepsilon}^{\prime}\left(v_{\varepsilon, \delta}\right) \frac{\partial v_{\varepsilon, \delta}}{\partial t}$ and noting $(2.5 \mathrm{c}),(2.15 \mathrm{a}),(2.15 \mathrm{~b})$ and the fact that $b_{\delta}^{\prime}, \psi_{\varepsilon}^{\prime} \leq C$.

It remains to bound $\int_{0}^{T}\left|\frac{\partial u_{\varepsilon}, \delta}{\partial t}\right|_{1}^{2} d t$. Differentiating (2.4a) with respect to time, noting that $\frac{\partial\left[f\left(u_{\varepsilon, \delta}\right)\right]}{\partial t}=f^{\prime}\left(u_{\varepsilon, \delta}\right) \frac{\partial u_{\varepsilon, \delta}}{\partial t}$ a.e. in $\Omega_{T}$, see e.g. Theorem 2.1.11 in Ziemer ${ }^{12}$; 
and multiplying by $\frac{\partial u_{\varepsilon, \delta}}{\partial t}$ we obtain that

$$
\begin{aligned}
\frac{1}{2} \frac{d}{d t}\left\|\frac{\partial u_{\varepsilon, \delta}}{\partial t}\right\|_{0}^{2}+c\left|\frac{\partial u_{\varepsilon, \delta}}{\partial t}\right|_{1}^{2} & =-\left(\frac{\partial u_{\varepsilon, \delta}}{\partial t}, f^{\prime}\left(u_{\varepsilon, \delta}\right) v_{\varepsilon, \delta} \frac{\partial u_{\varepsilon, \delta}}{\partial t}+f\left(u_{\varepsilon, \delta}\right) \frac{\partial v_{\varepsilon, \delta}}{\partial t}\right) \\
& \leq C\left\|\frac{\partial u_{\varepsilon, \delta}}{\partial t}\right\|_{0}^{2}+\left\|\frac{\partial v_{\varepsilon, \delta}}{\partial t}\right\|_{\left(H^{1}\right)^{\prime}}\left\|f\left(u_{\varepsilon, \delta}\right) \frac{\partial u_{\varepsilon, \delta}}{\partial t}\right\|_{1} \\
& \leq C\left\|\frac{\partial u_{\varepsilon, \delta}}{\partial t}\right\|_{0}^{2}+C\left(\left|u_{\varepsilon, \delta}\right|_{1, \infty}\left\|\frac{\partial u_{\varepsilon, \delta}}{\partial t}\right\|_{0}+\left|\frac{\partial u_{\varepsilon, \delta}}{\partial t}\right|_{1}\right) \\
& \leq \frac{c}{2}\left|\frac{\partial u_{\varepsilon, \delta}}{\partial t}\right|_{1}^{2}+C\left(1+\left|u_{\varepsilon, \delta}\right|_{1, \infty}^{2}\right)\left\|\frac{\partial u_{\varepsilon, \delta}}{\partial t}\right\|_{0}^{2}+C
\end{aligned}
$$

where we have used $(2.15 \mathrm{c})$ and $(2.5 \mathrm{c})$. The desired bound now follows from Gronwall's inequality, (2.15a) and the fact that $u^{0} \in H^{2}(\Omega)$ and $\frac{\partial u^{0}}{\partial \nu}=0$ on $\partial \Omega$.

Corollary 2.2. Let the assumptions of Theorem 2.1 hold. Then for $\varepsilon \in\left(0, \varepsilon_{1}\right]$ there exists a unique solution of

$\left(\boldsymbol{P}_{\varepsilon}\right)$ Find $\left(u_{\varepsilon}, v_{\varepsilon}\right)$ such that for a.a. $t \in(0, T)$

$$
\begin{aligned}
& \left(\frac{\partial u_{\varepsilon}}{\partial t}, \phi\right)+c\left(\nabla u_{\varepsilon}, \nabla \phi\right)=-\left(f\left(u_{\varepsilon}\right) v_{\varepsilon}, \phi\right) \quad \forall \phi \in H^{1}(\Omega), \\
& \left(\frac{\partial v_{\varepsilon}}{\partial t}, \phi\right)+\left(b\left(u_{\varepsilon}\right) \nabla\left[\psi_{\varepsilon}\left(v_{\varepsilon}\right)\right], \nabla \phi\right)=\theta\left(f\left(u_{\varepsilon}\right) v_{\varepsilon}, \phi\right) \quad \forall \phi \in H^{1}(\Omega)
\end{aligned}
$$

with $u_{\varepsilon}(\cdot, 0)=u^{0}(\cdot), v_{\varepsilon}(\cdot, 0)=v^{0}(\cdot)$.

Moreover $\left(u_{\varepsilon}, v_{\varepsilon}, w_{\varepsilon} \equiv \psi_{\varepsilon}\left(v_{\varepsilon}\right)\right)$ satisfy the following bounds:

$$
\begin{aligned}
& \sup _{t \in(0, T)}\left\|u_{\varepsilon}(\cdot, t)\right\|_{1, p^{*}}^{p^{*}}+\int_{0}^{T}\left(\left\|\frac{\partial u_{\varepsilon}}{\partial t}\right\|_{1}^{2}+\left\|u_{\varepsilon}\right\|_{2, p^{*}}^{p^{*}}+\left\|u_{\varepsilon}\right\|_{1, \infty}^{p^{*}}\right) d t \leq C, \\
& \sup _{t \in(0, T)}\left\|w_{\varepsilon}(\cdot, t)\right\|_{1}^{2}+\int_{0}^{T}\left\|\frac{\partial w_{\varepsilon}}{\partial t}\right\|_{0}^{2} d t \leq C, \\
& \sup _{t \in(0, T)}\left\|\frac{\partial v_{\varepsilon}}{\partial t}(\cdot, t)\right\|_{\left(H^{1}\right)^{\prime}}^{2}+\psi_{\varepsilon}^{\prime}(0) \int_{0}^{T}\left\|\frac{\partial v_{\varepsilon}}{\partial t}\right\|_{0}^{2} d t \leq C, \\
& 0 \leq u_{\varepsilon} \leq \bar{u}, \quad 0 \leq v_{\varepsilon} \leq v^{*} \quad \text { in } \Omega_{T} .
\end{aligned}
$$

Proof. Existence and the bounds (2.18a)-(2.18d) follow from letting $\delta \searrow 0$ in $\left(\mathrm{P}_{\varepsilon, \delta}\right)$ and in the bounds $(2.15 \mathrm{a})-(2.15 \mathrm{~d})$ and $(2.5 \mathrm{c})$, respectively. Since the corresponding arguments are simpler than those needed in passing to limit $\varepsilon \searrow 0$ in $\left(\mathrm{P}_{\varepsilon}\right)$ to establish existence of a weak solution to $(\mathrm{P})$, see the proof of Theorem 2.2 below; we omit the proof here. Similarly the uniqueness proof for $(\mathrm{P})$, given in Theorem 2.3 below, can easily be simplified to establish uniqueness for $\left(\mathrm{P}_{\varepsilon}\right)$.

\subsection{The Limit Problem}

We have the following existence result: 
Theorem 2.2. Let the stated assumptions on the data and coefficients in Section 1 hold. Then there exists a weak solution $(u, v)$ of $(P)$ such that

$$
\begin{aligned}
& u \in C^{0}\left(\bar{\Omega}_{T}\right) \cap L^{2}\left(0, T ; W^{1, \infty}(\Omega)\right) \cap L^{p^{*}}\left(0, T ; W^{2, p^{*}}(\Omega)\right), \quad \frac{\partial u}{\partial t} \in L^{2}\left(\Omega_{T}\right), \\
& v \in L^{\infty}\left(\Omega_{T}\right), \quad \psi(v) \in L^{\infty}\left(0, T ; H^{1}(\Omega)\right), \quad \frac{\partial v}{\partial t} \in L^{\infty}\left(0, T ;\left(H^{1}(\Omega)\right)^{\prime}\right), \\
& 0 \leq u \leq \bar{u}, \quad 0 \leq v \leq v^{*} \quad \text { in } \Omega_{T} ;
\end{aligned}
$$

and for a.a. $t \in(0, T)$

$$
\begin{aligned}
\left(\frac{\partial u}{\partial t}, \phi\right)+c(\nabla u, \nabla \phi)=-(f(u) v, \phi) & \forall \phi \in H^{1}(\Omega), \\
\left\langle\frac{\partial v}{\partial t}, \phi\right\rangle+(b(u) \nabla[\psi(v)], \nabla \phi)=\theta(f(u) v, \phi) & \forall \phi \in H^{1}(\Omega) \\
\text { with } u(\cdot, 0)=u^{0}(\cdot), v(\cdot, 0)=v^{0}(\cdot) . &
\end{aligned}
$$

Proof. We infer from (2.18a), (2.18b), (2.18d) and well-known compactness results that there exists $u \in C^{0}\left(\bar{\Omega}_{T}\right) \cap L^{2}\left(0, T ; W^{1, \infty}(\Omega)\right) \cap L^{p^{*}}\left(0, T ; W^{2, p^{*}}(\Omega)\right)$, $w \in L^{\infty}\left(\Omega_{T}\right)$ and a sequence $\varepsilon_{j} \searrow 0, j \rightarrow \infty$, such that

$$
\begin{array}{rlll}
u_{\varepsilon_{j}} & \rightarrow u & \text { uniformly on } \bar{\Omega}_{T}, \\
u_{\varepsilon_{j}} & \stackrel{*}{ } u & \text { in } L^{2}\left(0, T ; W^{1, \infty}(\Omega)\right), \\
\frac{\partial u_{\varepsilon_{j}}}{\partial t} & \rightarrow & \frac{\partial u}{\partial t} & \text { in } L^{2}\left(\Omega_{T}\right), \\
\psi_{\varepsilon_{j}}\left(v_{\varepsilon_{j}}\right) & \rightarrow w & \text { in } L^{2}\left(\Omega_{T}\right) \text { and a.e. in } \Omega_{T}, \\
\psi_{\varepsilon_{j}}\left(v_{\varepsilon_{j}}\right) & \stackrel{*}{ } w & \text { in } L^{\infty}\left(0, T ; H^{1}(\Omega)\right) .
\end{array}
$$

In addition, on noting (2.3) and as $\psi$ is strictly increasing, we deduce that $\psi^{-1}$ exists and hence that

$$
\begin{array}{rlll}
v_{\varepsilon_{j}} & \rightarrow \quad v:=\psi^{-1}(w) & & \text { a.e. in } \Omega_{T}, \\
\psi_{\varepsilon_{j}}\left(v_{\varepsilon_{j}}\right) & \stackrel{*}{\rightarrow} \psi(v) & & \text { in } L^{\infty}\left(0, T ; H^{1}(\Omega)\right) .
\end{array}
$$

Furthermore, (2.18c) yields, after possibly passing to another subsequence, that

$$
\frac{\partial v_{\varepsilon_{j}}}{\partial t} \quad \stackrel{*}{\rightarrow} \quad \frac{\partial v}{\partial t} \quad \text { in } L^{\infty}\left(0, T ;\left(H^{1}(\Omega)\right)^{\prime}\right) .
$$

Hence the relations (2.20a) $-(2.20 \mathrm{~b})$ and (2.19) follow from (2.4a) $-(2.4 \mathrm{~b}),(2.18 \mathrm{a})-$ (2.18d), (2.21)-(2.23) by sending $j \rightarrow \infty$. Finally, we have that $u(\cdot, 0)=u^{0}(\cdot)$ and $v(\cdot, 0)=v^{0}(\cdot)$. that

For the proof of our uniqueness result, we introduce $\mathcal{G}:\left(H^{1}(\Omega)\right)^{\prime} \rightarrow H^{1}(\Omega)$ such

$$
(\nabla \mathcal{G} z, \nabla \eta)+(\mathcal{G} z, \eta)=\langle z, \eta\rangle \quad \forall \eta \in H^{1}(\Omega)
$$


We note that $\|\mathcal{G} \cdot\|_{1} \equiv\|\cdot\|_{\left(H^{1}\right)^{\prime}} \cdot$

Theorem 2.3. Let the assumptions of Theorem 2.2 hold. Then the weak solution $(u, v)$ of $(P)$, (2.20a)-(2.20b), is unique.

Proof. Assume that there exist two solutions, $\left(u_{i}, v_{i}\right)$, to (P). Let $\hat{u}:=u_{1}-u_{2}$ and $\hat{v}:=v_{1}-v_{2}$. Then we have that

$$
\begin{aligned}
\left(\frac{\partial \hat{u}}{\partial t}, \hat{u}\right)+c|\hat{u}|_{1}^{2}= & -\left(f\left(u_{1}\right) v_{1}-f\left(u_{2}\right) v_{2}, \hat{u}\right) \\
\left\langle\frac{\partial \hat{v}}{\partial t}, \mathcal{G} \hat{v}\right\rangle+\left(\nabla\left[b\left(u_{1}\right) \psi\left(v_{1}\right)-b\left(u_{2}\right) \psi\left(v_{2}\right)\right],\right. & \nabla[\mathcal{G} \hat{v}]) \\
=\left(\nabla\left[b\left(u_{1}\right)\right] \psi\left(v_{1}\right)-\nabla\left[b\left(u_{2}\right)\right] \psi\left(v_{2}\right), \nabla[\mathcal{G} \hat{v}]\right) & +\theta\left(f\left(u_{1}\right) v_{1}-f\left(u_{2}\right) v_{2}, \mathcal{G} \hat{v}\right) .
\end{aligned}
$$

On rearranging $(2.25 \mathrm{a})-(2.25 \mathrm{~b})$ and noting $(2.24)$, we have that

$$
\begin{aligned}
& \frac{1}{2} \frac{d}{d t}\|\hat{u}\|_{0}^{2}+c|\hat{u}|_{1}^{2}+\left(v_{2}\left[f\left(u_{1}\right)-f\left(u_{2}\right)\right], \hat{u}\right)=-\left(f\left(u_{1}\right) \hat{v}, \hat{u}\right) \\
& \quad \leq\left\|f\left(u_{1}\right) \hat{u}\right\|_{1}\|\mathcal{G} \hat{v}\|_{1} \leq \frac{1}{2} c|\hat{u}|_{1}^{2}+\|\hat{u}\|_{0}^{2}+C\left(1+\left\|u_{1}\right\|_{1, \infty}^{2}\right)\|\mathcal{G} \hat{v}\|_{1}^{2},
\end{aligned}
$$

as well as

$$
\begin{aligned}
\frac{1}{2} \frac{d}{d t}\|\mathcal{G} \hat{v}\|_{1}^{2}+\left(b\left(u_{1}\right)\left[\psi\left(v_{1}\right)-\psi\left(v_{2}\right)\right], \hat{v}\right) \\
=\left(\left[b\left(u_{2}\right)-b\left(u_{1}\right)\right] \psi\left(v_{2}\right), \hat{v}\right)+\left(\left[b\left(u_{1}\right) \psi\left(v_{1}\right)-b\left(u_{2}\right) \psi\left(v_{2}\right)\right], \mathcal{G} \hat{v}\right) \\
\quad+\left(\nabla\left[b\left(u_{1}\right)\right] \psi\left(v_{1}\right)-\nabla\left[b\left(u_{2}\right)\right] \psi\left(v_{2}\right), \nabla[\mathcal{G} \hat{v}]\right) \\
\quad+\theta\left(f\left(u_{1}\right) v_{1}-f\left(u_{2}\right) v_{2}, \mathcal{G} \hat{v}\right) \\
=: \sum_{i=1}^{4} T_{i} .
\end{aligned}
$$

We now bound each of the terms $T_{i}$ on the right-hand-side of (2.27). Using Young's inequality, (1.2) and the boundedness of $u_{i}, v_{i}$ we infer for any $\gamma>0$ that

$$
\begin{aligned}
T_{1} & =-\left(b^{\prime}\left(u_{1}\right) \hat{u}, \psi\left(v_{2}\right) \hat{v}\right)-\int_{0}^{1}\left(\left[b^{\prime}\left(\tau u_{2}+(1-\tau) u_{1}\right)-b^{\prime}\left(u_{1}\right)\right] d \tau \hat{u}, \psi\left(v_{2}\right) \hat{v}\right) \\
& \leq \gamma \int_{\Omega} b\left(u_{1}\right)\left[\psi\left(v_{2}\right) \hat{v}\right]^{2}+C_{\gamma}\|\hat{u}\|_{0}^{2} .
\end{aligned}
$$

Let us focus on the product $\left[\psi\left(v_{2}\right) \hat{v}\right]^{2}$, which appears in the first term. Note first that $0 \leq v_{i} \leq v^{*}$ a.e. in $\Omega_{T}$ and let $v_{\min }:=\min \left(v_{1}, v_{2}\right)$. The convexity of $\psi$ implies that $\psi^{\prime}$ is increasing and together with $\psi(0)=0$ that $\psi(s) \leq s \psi^{\prime}(s)$, for $s \geq 0$. 
Then it follows that

$$
\begin{aligned}
{\left[\psi\left(v_{2}\right) \hat{v}\right]^{2} } & =\left(\left[\psi\left(v_{2}\right)\right]^{2}-\left[\psi\left(v_{\min }\right)\right]^{2}\right) \hat{v}^{2}+\left[\psi\left(v_{\min }\right) \hat{v}\right]^{2} \\
& \leq C\left(\psi\left(v_{2}\right)-\psi\left(v_{\min }\right)\right)\left(v_{2}-v_{\min }\right)+C \psi^{\prime}\left(v_{\min }\right) \hat{v}^{2} \\
& \leq C\left(\psi\left(v_{2}\right)-\psi\left(v_{1}\right)\right)\left(v_{2}-v_{1}\right)+C \hat{v} \int_{0}^{1} \psi^{\prime}\left(s v_{1}+(1-s) v_{2}\right) \hat{v} d s \\
& =C\left(\psi\left(v_{1}\right)-\psi\left(v_{2}\right)\right) \hat{v} .
\end{aligned}
$$

Thus we have that

$$
\int_{\Omega} b\left(u_{1}\right)\left[\psi\left(v_{2}\right) \hat{v}\right]^{2} \leq C\left(b\left(u_{1}\right)\left[\psi\left(v_{1}\right)-\psi\left(v_{2}\right)\right], \hat{v}\right),
$$

and hence

$$
T_{1} \leq \gamma\left(b\left(u_{1}\right)\left[\psi\left(v_{1}\right)-\psi\left(v_{2}\right)\right], \hat{v}\right)+C_{\gamma}\|\hat{u}\|_{0}^{2} .
$$

Next as $\psi$ is increasing and convex, it follows that

$$
\begin{aligned}
T_{2} & =\left(b\left(u_{1}\right)\left[\psi\left(v_{1}\right)-\psi\left(v_{2}\right)\right], \mathcal{G} \hat{v}\right)+\left(\left[b\left(u_{1}\right)-b\left(u_{2}\right)\right] \psi\left(v_{2}\right), \mathcal{G} \hat{v}\right) \\
& \leq \gamma \int_{\Omega} b\left(u_{1}\right)\left[\psi\left(v_{1}\right)-\psi\left(v_{2}\right)\right]^{2}+C_{\gamma}\|\mathcal{G} \hat{v}\|_{0}^{2}+C\|\hat{u}\|_{0}^{2} \\
& \leq C \gamma\left(b\left(u_{1}\right)\left[\psi\left(v_{1}\right)-\psi\left(v_{2}\right)\right], \hat{v}\right)+C_{\gamma}\|\mathcal{G} \hat{v}\|_{0}^{2}+C\|\hat{u}\|_{0}^{2} .
\end{aligned}
$$

Next we rewrite

$$
\begin{aligned}
T_{3}= & \left(b^{\prime}\left(u_{1}\right)\left[\psi\left(v_{1}\right)-\psi\left(v_{2}\right)\right] \nabla u_{1}, \nabla[\mathcal{G} \hat{v}]\right) \\
& \quad+\left(\left[b^{\prime}\left(u_{1}\right)-b^{\prime}\left(u_{2}\right)\right] \psi\left(v_{2}\right) \nabla u_{1}, \nabla[\mathcal{G} \hat{v}]\right)+\left(b^{\prime}\left(u_{2}\right) \psi\left(v_{2}\right) \nabla \hat{u}, \nabla[\mathcal{G} \hat{v}]\right) \\
= & : T_{3,1}+T_{3,2}+T_{3,3} .
\end{aligned}
$$

Firstly, (1.2) and (2.19) imply that

$$
\begin{aligned}
T_{3,1} & \leq \gamma \int_{\Omega} b\left(u_{1}\right)\left[\psi\left(v_{1}\right)-\psi\left(v_{2}\right)\right]^{2}+C_{\gamma}\left\|\nabla u_{1}\right\|_{0, \infty}^{2}\|\nabla[\mathcal{G} \hat{v}]\|_{0}^{2} \\
& \leq C \gamma\left(b\left(u_{1}\right)\left[\psi\left(v_{1}\right)-\psi\left(v_{2}\right)\right], \hat{v}\right)+C_{\gamma}\left\|\nabla u_{1}\right\|_{0, \infty}^{2}\|\nabla[\mathcal{G} \hat{v}]\|_{0}^{2},
\end{aligned}
$$

while

$$
\begin{aligned}
T_{3,2}+T_{3,3} & \leq C\|\hat{u}\|_{0}\left\|\nabla u_{1}\right\|_{0, \infty}\|\nabla[\mathcal{G} \hat{v}]\|_{0}+C\|\nabla \hat{u}\|_{0}\|\nabla[\mathcal{G} \hat{v}]\|_{0} \\
& \leq \gamma\|\hat{u}\|_{1}^{2}+C_{\gamma}\left(1+\left\|\nabla u_{1}\right\|_{0, \infty}^{2}\right)\|\nabla[\mathcal{G} \hat{v}]\|_{0}^{2} .
\end{aligned}
$$

In conclusion, we obtain that

$$
T_{3} \leq C \gamma\left(b\left(u_{1}\right)\left[\psi\left(v_{1}\right)-\psi\left(v_{2}\right)\right], \hat{v}\right)+\gamma\|\hat{u}\|_{1}^{2}+C_{\gamma}\left(1+\left\|\nabla u_{1}\right\|_{0, \infty}^{2}\right)\|\mathcal{G} \hat{v}\|_{1}^{2} .
$$

Finally, we have on noting (2.24) that

$$
\begin{aligned}
T_{4} & =\theta\left(\left[f\left(u_{1}\right)-f\left(u_{2}\right)\right] v_{2}, \mathcal{G} \hat{v}\right)+\theta\left(f\left(u_{1}\right) \hat{v}, \mathcal{G} \hat{v}\right) \\
& \leq C\|\hat{u}\|_{0}\|\mathcal{G} \hat{v}\|_{0}+\|\mathcal{G} \hat{v}\|_{1}\left\|f\left(u_{1}\right) \mathcal{G} \hat{v}\right\|_{1} \\
& \leq\|\hat{u}\|_{0}^{2}+C\left(1+\left\|u_{1}\right\|_{1, \infty}^{2}\right)\|\mathcal{G} \hat{v}\|_{1}^{2} .
\end{aligned}
$$


Combining (2.26)-(2.31), we obtain after choosing $\gamma$ sufficiently small that

$$
\begin{aligned}
& \frac{d}{d t}\left(\|\hat{u}\|_{0}^{2}+\|\mathcal{G} \hat{v}\|_{1}^{2}\right)+2 c|\hat{u}|_{1}^{2}+2\left(b\left(u_{1}\right)\left[\psi\left(v_{1}\right)-\psi\left(v_{2}\right)\right], \hat{v}\right) \\
& \quad \leq\left(b\left(u_{1}\right)\left[\psi\left(v_{1}\right)-\psi\left(v_{2}\right)\right], \hat{v}\right)+c|\hat{u}|_{1}^{2}+C\|\hat{u}\|_{0}^{2}+C\left(1+\left\|u_{1}\right\|_{1, \infty}^{2}\right)\|\mathcal{G} \hat{v}\|_{1}^{2} .
\end{aligned}
$$

A Gronwall inequality, on noting (2.19), now implies that $\hat{u}=\hat{v}=0$, and hence uniqueness.

Theorem 2.4. Under the assumptions of Theorem 2.2, we have the following regularization error bound between the unique solutions $(u, v)$ of $(P)$ and $\left(u_{\varepsilon}, v_{\varepsilon}\right)$ of $\left(P_{\varepsilon}\right), \varepsilon \in\left(0, \varepsilon_{1}\right]:$

$$
\begin{gathered}
\sup _{t \in(0, T)}\left\|\left(u-u_{\varepsilon}\right)(\cdot, t)\right\|_{0}^{2}+\int_{0}^{T}\left|u-u_{\varepsilon}\right|_{1}^{2} d t+\sup _{t \in(0, T)}\left\|\left[\mathcal{G}\left(v-v_{\varepsilon}\right)\right](\cdot, t)\right\|_{1}^{2} \\
+\int_{0}^{T}\left(b(u)\left[\psi_{\varepsilon}(v)-\psi_{\varepsilon}\left(v_{\varepsilon}\right)\right], v-v_{\varepsilon}\right) d t \leq C \varepsilon q_{\varepsilon}^{2},
\end{gathered}
$$

where $q_{\varepsilon}$ is as defined for (2.2).

Proof. The proof is a simple adaptation of the uniqueness proof, Theorem 2.3, above. Let $\hat{u}_{\varepsilon}:=u-u_{\varepsilon}$ and $\hat{v}_{\varepsilon}:=v-v_{\varepsilon}$. Then, similarly to (2.26) and (2.27), we have on noting $(2.20 \mathrm{a})-(2.20 \mathrm{~b}),(2.17 \mathrm{a})-(2.17 \mathrm{~b})$ and $(2.24)$ that

$$
\begin{gathered}
\frac{1}{2} \frac{d}{d t}\left\|\hat{u}_{\varepsilon}\right\|_{0}^{2}+c\left|\hat{u}_{\varepsilon}\right|_{1}^{2}+\left(v_{\varepsilon}\left[f(u)-f\left(u_{\varepsilon}\right)\right], \hat{u}_{\varepsilon}\right)=-\left(f(u) \hat{v}_{\varepsilon}, \hat{u}_{\varepsilon}\right) \\
\leq \frac{1}{2} c\left|\hat{u}_{\varepsilon}\right|_{1}^{2}+\left\|\hat{u}_{\varepsilon}\right\|_{0}^{2}+C\left(1+\|u\|_{1, \infty}^{2}\right)\left\|\mathcal{G} \hat{v}_{\varepsilon}\right\|_{1}^{2},
\end{gathered}
$$

and

$$
\begin{aligned}
& \frac{1}{2} \frac{d}{d t}\left\|\mathcal{G} \hat{v}_{\varepsilon}\right\|_{1}^{2}+\left(b(u)\left[\psi_{\varepsilon}(v)-\psi_{\varepsilon}\left(v_{\varepsilon}\right)\right], \hat{v}_{\varepsilon}\right) \\
&=\left(\left[b\left(u_{\varepsilon}\right)-b(u)\right] \psi_{\varepsilon}\left(v_{\varepsilon}\right), \hat{v}_{\varepsilon}\right)+\left(\left[b(u) \psi(v)-b\left(u_{\varepsilon}\right) \psi_{\varepsilon}\left(v_{\varepsilon}\right)\right], \mathcal{G} \hat{v}_{\varepsilon}\right) \\
& \quad+\left(\nabla[b(u)] \psi(v)-\nabla\left[b\left(u_{\varepsilon}\right)\right] \psi_{\varepsilon}\left(v_{\varepsilon}\right), \nabla\left[\mathcal{G} \hat{v}_{\varepsilon}\right]\right) \\
& \quad+\theta\left(f(u) v-f\left(u_{\varepsilon}\right) v_{\varepsilon}, \mathcal{G} \hat{v}_{\varepsilon}\right)+\left(b(u)\left[\psi_{\varepsilon}(v)-\psi(v)\right], \hat{v}_{\varepsilon}\right) \\
&=: \sum_{i=1}^{5} T_{i}^{\varepsilon} .
\end{aligned}
$$

We now bound each of the terms $T_{i}^{\varepsilon}$ on the right-hand-side of (2.34). The first term $T_{1}^{\varepsilon}$ is bounded as $T_{1}$, and we have the following analogue of (2.28) for any $\gamma>0$

$$
T_{1}^{\varepsilon} \leq \gamma\left(b(u)\left[\psi_{\varepsilon}(v)-\psi_{\varepsilon}\left(v_{\varepsilon}\right)\right], \hat{v}_{\varepsilon}\right)+C_{\gamma}\left\|\hat{u}_{\varepsilon}\right\|_{0}^{2} .
$$

Next, we have on noting (2.3) that

$$
\begin{aligned}
T_{2}^{\varepsilon}=\left(b(u)\left[\psi(v)-\psi_{\varepsilon}(v)\right]+b(u)\left[\psi_{\varepsilon}(v)-\psi_{\varepsilon}\left(v_{\varepsilon}\right)\right], \mathcal{G} \hat{v}_{\varepsilon}\right) & \\
& +\left(\left[b(u)-b\left(u_{\varepsilon}\right)\right] \psi_{\varepsilon}\left(v_{\varepsilon}\right), \mathcal{G} \hat{v}_{\varepsilon}\right) \\
\leq C \gamma\left(b(u)\left[\psi_{\varepsilon}(v)-\psi_{\varepsilon}\left(v_{\varepsilon}\right)\right], \hat{v}_{\varepsilon}\right) & +C_{\gamma}\left\|\mathcal{G} \hat{v}_{\varepsilon}\right\|_{0}^{2}+C\left[\left\|\hat{u}_{\varepsilon}\right\|_{0}^{2}+\left(\varepsilon q_{\varepsilon}\right)^{2}\right] .
\end{aligned}
$$


We have also that

$$
\begin{aligned}
T_{3}^{\varepsilon}=\left(b^{\prime}(u)\left[\psi_{\varepsilon}(v)-\psi_{\varepsilon}\left(v_{\varepsilon}\right)\right] \nabla u, \nabla\left[\mathcal{G} \hat{v}_{\varepsilon}\right]\right)+\left(\left[b^{\prime}(u)-b^{\prime}\left(u_{\varepsilon}\right)\right] \psi_{\varepsilon}\left(v_{\varepsilon}\right) \nabla u, \nabla\left[\mathcal{G} \hat{v}_{\varepsilon}\right]\right) \\
+\left(b^{\prime}\left(u_{\varepsilon}\right) \psi_{\varepsilon}\left(v_{\varepsilon}\right) \nabla \hat{u}_{\varepsilon}, \nabla\left[\mathcal{G} \hat{v}_{\varepsilon}\right]\right)+\left(\left[\psi(v)-\psi_{\varepsilon}(v)\right] \nabla[b(u)], \nabla\left[\mathcal{G} \hat{v}_{\varepsilon}\right]\right) \\
=: T_{3,1}^{\varepsilon}+T_{3,2}^{\varepsilon}+T_{3,3}^{\varepsilon}+T_{3,4}^{\varepsilon} .
\end{aligned}
$$

Bounding $T_{3, i}^{\varepsilon}, i=1 \rightarrow 4$, as $T_{3, i}$ in (2.30) yields that

$$
\begin{aligned}
T_{3}^{\varepsilon} \leq C \gamma\left(b(u)\left[\psi_{\varepsilon}(v)-\psi_{\varepsilon}\left(v_{\varepsilon}\right)\right], \hat{v}_{\varepsilon}\right)+\gamma\left\|\hat{u}_{\varepsilon}\right\|_{1}^{2} \\
+C_{\gamma}\left(1+\|\nabla u\|_{0, \infty}^{2}\right)\left\|\mathcal{G} \hat{v}_{\varepsilon}\right\|_{1}^{2}+C\left(\varepsilon q_{\varepsilon}\right)^{2} .
\end{aligned}
$$

Next bounding $T_{4}^{\varepsilon}$ in an analogous way to $T_{4}$ in (2.31), we obtain that

$$
T_{4}^{\varepsilon} \leq\left\|\hat{u}_{\varepsilon}\right\|_{0}^{2}+C\left(1+\|u\|_{1, \infty}^{2}\right)\left\|\mathcal{G} \hat{v}_{\varepsilon}\right\|_{1}^{2} .
$$

Finally, we have on noting (2.3) that

$$
\begin{aligned}
T_{5}^{\varepsilon} & =\left(b(u)\left[\psi_{\varepsilon}(v)-\psi(v)\right], \hat{v}_{\varepsilon}\right) \leq C \varepsilon q_{\varepsilon}\left(b(u) \hat{v}_{\varepsilon}, \hat{v}_{\varepsilon}\right)^{\frac{1}{2}} \\
& \leq \gamma\left(b(u)\left[\psi_{\varepsilon}(v)-\psi_{\varepsilon}\left(v_{\varepsilon}\right)\right], \hat{v}_{\varepsilon}\right)+C_{\gamma} \varepsilon q_{\varepsilon}^{2} .
\end{aligned}
$$

Combining (2.33)-(2.39), we obtain after choosing $\gamma$ sufficiently small that

$$
\begin{aligned}
\frac{d}{d t}\left(\left\|\hat{u}_{\varepsilon}\right\|_{0}^{2}+\left\|\mathcal{G} \hat{v}_{\varepsilon}\right\|_{1}^{2}\right)+2 c\left|\hat{u}_{\varepsilon}\right|_{1}^{2}+ & 2\left(b(u)\left[\psi_{\varepsilon}(v)-\psi_{\varepsilon}\left(v_{\varepsilon}\right)\right], \hat{v}_{\varepsilon}\right) \\
\leq\left(b ( u ) \left[\psi_{\varepsilon}(v)-\right.\right. & \left.\left.\psi_{\varepsilon}\left(v_{\varepsilon}\right)\right], \hat{v}_{\varepsilon}\right)+c\left|\hat{u}_{\varepsilon}\right|_{1}^{2}+C\left\|\hat{u}_{\varepsilon}\right\|_{0}^{2} \\
& +C\left(1+\|u\|_{1, \infty}^{2}\right)\left\|\mathcal{G} \hat{v}_{\varepsilon}\right\|_{1}^{2}+C \varepsilon q_{\varepsilon}^{2} .
\end{aligned}
$$

A Gronwall inequality, on noting (2.19), now implies the desired result (2.32).

Remark 2.1. In Theorem 1 of Nochetto and Verdi, ${ }^{8}$ a regularization error bound is proved for the porous medium equation; that is, (1.1b) with $b(u) \equiv 1$ and a more general right-hand-side. The regularization of $\psi$ in Nochetto and Verdi ${ }^{8}$ is the same as $(2.2)$; but with $q_{\varepsilon}$ replaced by $\widehat{q}_{\varepsilon}$, and with $\psi_{\varepsilon}(s) \equiv \psi(s)$ for $s \geq \widehat{q}_{\varepsilon}$, leading to $\psi_{\varepsilon} \in C_{\text {loc }}^{0,1}\left(\mathbb{R}_{\geq 0}\right)$. Here $\widehat{q}_{\varepsilon}$ is the maximal solution of $\varepsilon s=\psi(s)$. It is easily deduced that $\widehat{q}_{\varepsilon} \geq q_{\varepsilon}$. The error bound in Nochetto and Verdi ${ }^{8}$ for this single degenerate equation is the same as for the terms involving $v$ in (2.32), but with $q_{\varepsilon}$ replaced by $\widehat{q}_{\varepsilon}, b(u) \equiv 1$ and with the right-hand-side multiplied by

$$
A_{\varepsilon}:=\left\{(x, t) \in \Omega_{T}:\left[\psi(v)-\psi_{\varepsilon}(v)\right](x, t) \neq 0\right\} \equiv\left\{(x, t) \in \Omega_{T}: v(x, t) \in\left[0, \widehat{q}_{\varepsilon}\right)\right\} .
$$

Our analysis in the presence of the possibly degenerate $b(u)$ requires $\psi_{\varepsilon} \in C^{1}\left(\mathbb{R}_{\geq 0}\right)$ as in (2.2), and hence the absence of the factor $A_{\varepsilon}$ in (2.32). Nevertheless, it is remarkable that the bound (2.32) can be obtained for our possibly doubly-degenerate system $(\mathrm{P})$. 


\section{Finite Element Approximation}

We consider the finite element approximation of $(\mathrm{P})$ under the following assumptions on the mesh:

(A) We now restrict ourselves to the physically relevant case of $d \leq 3$. Let $\left\{\mathcal{T}^{h}\right\}_{h>0}$ regular family of partitionings of $\Omega^{h}$, a polyhedral approximation of $\Omega$ into disjoint open simplices $\kappa$ with $h_{\kappa}:=\operatorname{diam}(\kappa)$ and $h:=$ $\max _{\kappa \in \mathcal{T} h} h_{\kappa}$, so that $\overline{\Omega^{h}}=\cup_{\kappa \in \mathcal{T} h} \bar{\kappa}$. We assume that vertices lying on $\partial \Omega^{h}$, also lie on $\partial \Omega$. In addition, we assume that this partitioning is acute; that is, the angle between any two sides if $d=2$ (faces if $d=3$ ) of any simplex does not exceed $\frac{\pi}{2}$.

Associated with $\mathcal{T}^{h}$ is the finite element space

$$
S^{h}:=\left\{\chi \in C\left(\overline{\Omega^{h}}\right):\left.\chi\right|_{\kappa} \text { is affine } \forall \kappa \in \mathcal{T}^{h}\right\} \subset H^{1}\left(\Omega^{h}\right) .
$$

We introduce also $S_{\geq 0}^{h}:=\left\{\chi \in S^{h}: \chi \geq 0\right.$ in $\left.\Omega^{h}\right\}$. Let $J$ be the set of nodes of $\mathcal{T}^{h}$ and $\left\{p_{j}\right\}_{j \in J}$ the coordinates of these nodes. Let $\mathcal{J}:=\# J$ and $\left\{\chi_{j}\right\}_{j \in J}$ be the standard basis functions for $S^{h}$; that is $\chi_{j} \in S^{h}$ and $\chi_{j}\left(p_{i}\right)=\delta_{i j}$ for all $i, j \in J$. We introduce $I^{h}: C\left(\overline{\Omega^{h}}\right) \rightarrow S^{h}$, the interpolation operator, such that $I^{h} \eta\left(p_{j}\right)=\eta\left(p_{j}\right)$ for all $j \in J$. We require also the local interpolation operator $\left.I_{\kappa}^{h} \equiv I^{h}\right|_{\kappa}$ on each $\kappa \in \mathcal{T}^{h}$. A discrete inner product is then defined by

$$
\left(\eta_{1}, \eta_{2}\right)^{h}:=\sum_{\kappa \in \mathcal{T}^{h}} \int_{\kappa} I_{\kappa}^{h}\left(\eta_{1} \eta_{2}\right)
$$

where $\eta_{i}$ are piecewise continuous functions on the partitioning $\mathcal{T}^{h}$.

In general $\Omega \not \subset \Omega^{h}$. Therefore, in order to project the initial data and to analyse the error in our approximation $\left(\mathrm{P}_{\varepsilon}^{h, \tau}\right)$; we need to extend any $\chi \in S^{h}$, which is defined on $\Omega^{h}$, to $\Omega$. This we do in the standard way, see e.g. Nochetto and Verdi ${ }^{8}$. First let $\widetilde{\Omega}^{h}$ be the union of $\overline{\Omega^{h}}$ and those simplices, which are reflections across $\partial \Omega^{h}$ of simplices with one side on $\partial \Omega^{h}$ ("external simplices"). As $\partial \Omega \in C^{2}$, we have that $\Omega \subset \widetilde{\Omega}^{h}$ for all $h \leq h_{0}$. We then set

$$
\begin{array}{r}
\widetilde{S}^{h}:=\left\{\chi \in C\left(\widetilde{\Omega}^{h}\right):\left.\chi\right|_{\Omega^{h}} \in S^{h} \text { and } \chi\right. \text { is affine in the union of any } \\
\text { external } \kappa \text { with its corresponding reflected element }\} .
\end{array}
$$

Hence any $\chi \in S^{h}$ can be naturally extended to $\widetilde{S}^{h}$. As no confusion can arise, we will not distinguish between the elements of $S^{h}$ and $\widetilde{S}^{h}$.

Noting the above extension, we now introduce the $L^{2}$ projection $Q^{h}: L^{2}(\Omega) \rightarrow$ $S^{h}$ defined by

$$
\left(Q^{h} \eta, \chi\right)^{h}=(\eta, \chi) \quad \forall \chi \in S^{h} .
$$

In addition to $\mathcal{T}^{h}$, let $0 \equiv t_{0}<t_{1}<\ldots<t_{N-1}<t_{N} \equiv T$ be a partitioning of $[0, T]$ into possibly variable time steps $\tau_{n}:=t_{n}-t_{n-1}, n=1, \ldots, N$. We set $\tau:=\max _{n=1, \ldots, N} \tau_{n}, \tau_{\min }:=\min _{n=1, \ldots, N} \tau_{n}$ and define $d_{t} z^{n}:=\frac{z^{n}-z^{n-1}}{\tau_{n}}$. We then consider 
the following fully practical finite element approximation of $\left(\mathrm{P}_{\varepsilon}\right)$, and hence $(\mathrm{P})$ : $\left(\mathbf{P}_{\varepsilon}^{h, \tau}\right)$ For $n \geq 1$, find $\left\{U_{\varepsilon}^{n}, V_{\varepsilon}^{n}, W_{\varepsilon}^{n}\right\} \in\left[S^{h}\right]^{3}$ such that $W_{\varepsilon}^{n}\left(p_{j}\right)=\psi_{\varepsilon}\left(V_{\varepsilon}^{n}\left(p_{j}\right)\right)$ for all $j \in J$ and

$$
\begin{array}{ll}
\left(d_{t} U_{\varepsilon}^{n}, \chi\right)^{h}+c\left(\nabla U_{\varepsilon}^{n}, \nabla \chi\right)^{h}+\left(f\left(U_{\varepsilon}^{n}\right) V_{\varepsilon}^{n-1}, \chi\right)^{h}=0 & \forall \chi \in S^{h}, \\
\left(d_{t} V_{\varepsilon}^{n}, \chi\right)^{h}+\left(b\left(U_{\varepsilon}^{n}\right) \nabla W_{\varepsilon}^{n}, \nabla \chi\right)^{h}=\theta\left(f\left(U_{\varepsilon}^{n}\right) V_{\varepsilon}^{n-1}, \chi\right)^{h} & \forall \chi \in S^{h} ;
\end{array}
$$

where $U_{\varepsilon}^{0}, V_{\varepsilon}^{0} \in S_{>0}^{h}$ are approximations of $u^{0}, v^{0} \geq 0$, respectively, e.g. $U_{\varepsilon}^{0} \equiv I^{h} u^{0}$ and $V_{\varepsilon}^{0} \equiv Q^{h} v^{0}$. In fact, it is a simple matter to deduce that a solution of $\left(\mathrm{P}_{\varepsilon}^{h, \tau}\right)$ is such that $\left\{U_{\varepsilon}^{n}, V_{\varepsilon}^{n}, W_{\varepsilon}^{n}\right\} \in S_{\geq 0}^{h}, n=1, \ldots, N$, see (3.20a)-(3.20b) below.

Remark 3.1. We note that (3.4a) is independent of $V_{\varepsilon}^{n}$; and as the nonlinearity is diagonal, it is an almost linear system. ${ }^{9}$ Similarly, (3.4b) is an almost linear system for $W_{\varepsilon}^{n}$. Hence these decoupled systems are easily solved. Numerical experiments for a related, but slightly more complicated scheme can be found in Barrett and Nürnberg. ${ }^{1}$

Below we recall some well-known results concerning $S^{h}$ for any $\kappa \in \mathcal{T}^{h}, \chi, z^{h} \in$ $S^{h}, m \in\{0,1\}, p \in[1, \infty]$ and $q \in[2, \infty]:$

$$
\begin{aligned}
|\chi|_{1, p, \kappa} & \leq C h_{\kappa}^{-1}\|\chi\|_{0, p, \kappa}, \\
\left|\left(I-I_{\kappa}^{h}\right) \eta\right|_{m, q, \kappa} & \leq C h^{2-m}|\eta|_{2, q, \kappa} \\
\int_{\kappa}|\chi|^{2} \mathrm{~d} x & \leq \int_{\kappa} I^{h}\left[|\chi|^{2}\right] \mathrm{d} x \leq C \int_{\kappa}|\chi|^{2} \mathrm{~d} x,
\end{aligned} \quad \forall \eta \in W^{2, q}(\kappa),
$$

where $I$ in $(3.5 \mathrm{~b})$ denotes here, and throughout, the identity operator. We note that the acuteness assumption implies that

$$
\int_{\kappa} \nabla \chi_{i} \cdot \nabla \chi_{j} \leq 0 \quad i \neq j, \quad \forall \kappa \in \mathcal{T}^{h} ;
$$

which yields for monotone $g \in C^{0,1}(\mathbb{R})$ with Lipschitz constant $L_{g}$ that

$$
\int_{\kappa}\left|\nabla I^{h}[g(\chi)]\right|^{2} \leq L_{g} \int_{\kappa} \nabla \chi \cdot \nabla I^{h}[g(\chi)] \quad \forall \chi \in S^{h}, \quad \forall \kappa \in \mathcal{T}^{h} .
$$

Furthermore, it is easily established, see e.g. p69 in Elliott, ${ }^{3}$ that for all $\kappa \in \mathcal{T}^{h}$ and for all $\chi \in S^{h}$

$$
\left\|\left(I-I_{\kappa}^{h}\right)[g(\chi)]\right\|_{0, \infty, \kappa} \leq C h_{\kappa}\left|I_{\kappa}^{h}[g(\chi)]\right|_{1, \infty, \kappa} .
$$

In addition, we have for any piecewise quadratic function $\eta^{h}$, defined on $\widetilde{\Omega}^{h}$, that

$$
\int_{\left(\Omega \backslash \Omega^{h}\right) \cup\left(\Omega^{h} \backslash \Omega\right)}\left|\eta^{h}\right| \leq C h \int_{\widetilde{\Omega}^{h}}\left|\eta^{h}\right| ;
$$

see e.g. Lemma 2 in Nochetto and Verdi. ${ }^{8}$ It follows immediately from (3.2), (3.1) and (3.9) that for $m \in\{0,1\}, p \in[1, \infty]$ and any $\chi, z^{h} \in S^{h}$

$$
\begin{aligned}
C_{1}|\chi|_{m, p, \Omega^{h}} & \leq|\chi|_{m, p} \leq C_{2}|\chi|_{m, p, \Omega^{h},}, \\
\left|\left(\chi, z^{h}\right)-\left(\chi, z^{h}\right)^{h}\right| & \leq C h\|\chi\|_{1, p, \Omega^{h}}\left\|z^{h}\right\|_{0, p^{\prime}, \Omega^{h}},
\end{aligned}
$$


where $\frac{1}{p}+\frac{1}{p^{\prime}}=1$. Furthermore, it is easily deduced for $m \in\{0,1\}$ that

$$
\left\|\left(I-I^{h}\right) z\right\|_{m} \leq C h^{2-m}\|z\|_{2} \quad \text { and } \quad\left\|\mathcal{G}\left[\left(I-Q^{h}\right) z\right]\right\|_{1} \leq C h\|z\|_{0} .
$$

Next we introduce the $S^{h}$ approximation of $\mathcal{G}$ (recall $\left.(2.24)\right): \mathcal{G}^{h}:\left(H^{1}(\Omega)\right)^{\prime}$ $\rightarrow S^{h}$ such that

$$
\left(\nabla \mathcal{G}^{h} z, \nabla \chi\right)+\left(\mathcal{G}^{h} z, \chi\right)=\langle z, \chi\rangle \quad \forall \chi \in S^{h} .
$$

Moreover, we introduce the $S^{h}$ Ritz projection $\mathcal{R}^{h}: H^{1}(\Omega) \rightarrow S^{h}$ such that

$$
\left(\nabla\left[\left(I-\mathcal{R}^{h}\right) z\right], \nabla \chi\right)+\left(\left(I-\mathcal{R}^{h}\right) z, \chi\right)=0 \quad \forall \chi \in S^{h} ;
$$

and hence $\mathcal{G}^{h} z \equiv \mathcal{R}^{h}(\mathcal{G} z)$. We note that $\mathcal{G}^{h}$ and $\mathcal{R}^{h}$ are only required for the error analysis, and not required in practice. Therefore the integrals in (3.12) and (3.13) are defined over $\Omega$, and not $\Omega^{h}$. In which case, it is easily deduced, ${ }^{8}$ as $\partial \Omega \in C^{2}$, that for $m \in\{0,1\}$

$$
\begin{aligned}
& \left|\left(\mathcal{G}-\mathcal{G}^{h}\right) z\right|_{m} \leq C h^{1-m}\|\mathcal{G} z\|_{1}, \\
& \left|\left(\mathcal{G}-\mathcal{G}^{h}\right) z\right|_{m} \leq C h^{2-m}\|\mathcal{G} z\|_{2} \leq C h^{2-m}|z|_{0}, \\
\text { and } \quad & \left|\left(I-\mathcal{R}^{h}\right) z\right|_{m} \leq C h^{r-m}\|z\|_{r}, \quad \text { for } \quad r=1 \text { and } 2 .
\end{aligned}
$$

In order to bound a key term in the error analysis, we introduce the following matrices $D(\cdot)$. Let $\left\{e_{i}\right\}_{i=1}^{d}$ be the orthonormal vectors in $\mathbb{R}^{d}$, such that the $j^{\text {th }}$ component of $e_{i}$ is $\delta_{i j}, i, j=1 \rightarrow d$. Let $\widehat{\kappa}$ be the standard reference simplex in $\mathbb{R}^{d}$ with vertices $\left\{\widehat{p}_{i}\right\}_{i=0}^{d}$, where $\widehat{p}_{0}$ is the origin and $\widehat{p}_{i}=e_{i}, i=1 \rightarrow d$. Given a $\kappa \in \mathcal{T}^{h}$ with vertices $\left\{p_{i}\right\}_{i=0}^{d}$ there exists a matrix $B_{\kappa}$ such that the mapping $\mathcal{F}_{\kappa}: \widehat{x} \in \mathbb{R}^{d} \rightarrow b_{\kappa}+B_{\kappa} \widehat{x} \in \mathbb{R}^{d}$ maps the vertex $\widehat{p}_{i}$ to $p_{i}, i=0 \rightarrow d$, and hence $\widehat{\kappa}$ to $\kappa$. It is easily deduced, see p638 in Barrett and Nürnberg ${ }^{2}$ for details, that for all $\kappa \in \mathcal{T}^{h}$ and $\eta_{j} \in C(\bar{\kappa})$

$$
\nabla\left(I_{\kappa}^{h}\left[\eta_{1} \eta_{2}\right]\right)=D\left(I_{\kappa}^{h} \eta_{1}\right) \nabla\left(I_{\kappa}^{h} \eta_{2}\right)+D\left(I_{\kappa}^{h} \eta_{2}\right) \nabla\left(I_{\kappa}^{h} \eta_{1}\right) \quad \text { on } \kappa ;
$$

where for any $z^{h} \in S^{h}$

$$
\left.D\left(z^{h}\right)\right|_{\kappa}:=B_{\kappa}^{-T} \widehat{D}\left(z^{h}\right) B_{\kappa}^{T} \quad \forall \kappa \in \mathcal{T}^{h},
$$

and $\widehat{D}\left(z^{h}\right)$ is the $d \times d$ diagonal matrix with diagonal entries

$$
\left[\widehat{D}\left(z^{h}\right)\right]_{i i}:=\frac{1}{2}\left[z^{h}\left(p_{0}\right)+z^{h}\left(p_{i}\right)\right] \quad i=1 \rightarrow d .
$$

Furthermore, we have for all $z^{h} \in S^{h}$ and for all $\kappa \in \mathcal{T}^{h}$ that

$$
\left\|D\left(z^{h}\right)-z^{h} \mathcal{I}\right\|_{0, \infty, \kappa} \leq C h_{\kappa}\left\|\nabla z^{h}\right\|_{0, \infty, \kappa},
$$

where $\mathcal{I}$ is the $d \times d$ identity matrix.

Finally, we recall the elementary identity

$$
2 r(r-s)=\left(r^{2}-s^{2}\right)+(r-s)^{2} \quad \forall r, s \in \mathbb{R} .
$$

In addition, on introducing $\Psi_{\varepsilon}(s)=\int_{0}^{s} \psi_{\varepsilon}(r) d r$, we have that

$$
(r-s) \psi_{\varepsilon}(r) \geq \Psi_{\varepsilon}(r)-\Psi_{\varepsilon}(s) \quad \forall r, s \in \mathbb{R} .
$$




\subsection{Stability Bounds}

Theorem 3.1. Let the stated assumptions on the data and coefficients in Section 1 hold. Let the assumptions (A) hold, and $\left\{U_{\varepsilon}^{n-1}, V_{\varepsilon}^{n-1}\right\} \in\left[S_{>0}^{h}\right]^{2}$. Then for all $h \leq h_{0}$ and for all $\varepsilon, \tau_{n}>0$ there exists a unique solution $\left\{U_{\varepsilon}^{n}, V_{\varepsilon}^{n}\right\} \in S_{\geq 0}^{h}$ to the $n$-th step of $\left(P_{\varepsilon}^{h, \tau}\right)$.

Moreover, if $U_{\varepsilon}^{0}(x) \in\left[0, \mathcal{U}_{M}^{0}\right]$ and $V_{\varepsilon}^{0}(x) \in\left[\mathcal{V}_{m}^{0}, \mathcal{V}_{M}^{0}\right]$ for all $x \in \overline{\Omega^{h}}$, where $\mathcal{U}_{M}^{0}, \mathcal{V}_{m}^{0}, \mathcal{V}_{M}^{0} \in \mathbb{R}_{\geq 0}$; then for $n=1 \rightarrow N$ it follows that

$$
\begin{aligned}
& \mathcal{U}_{M}^{0} \geq U_{\varepsilon}^{n}(x) \geq 0 \\
& \mathcal{V}_{M} \geq V_{\varepsilon}^{n}(x) \geq \mathcal{V}_{m}^{0}
\end{aligned}
$$

for all $x \in \overline{\Omega^{h}}$, where

$$
\mathcal{V}_{M}:=\exp \left(\theta f\left(\mathcal{U}_{M}^{0}\right) T\right) \mathcal{V}_{M}^{0} .
$$

Furthermore, $U_{\varepsilon}^{0} \equiv I^{h} u^{0}$ and $V_{\varepsilon}^{0} \equiv Q^{h} v^{0}$ yield that $\mathcal{U}_{m}^{0}=\mathcal{V}_{m}^{0}=0, \mathcal{U}_{M}^{0}=\bar{u}$ and $\mathcal{V}_{M}^{0} \leq(1+C h) \bar{v}$, and hence for $n=1 \rightarrow N$ that

$$
\begin{aligned}
\bar{u} & \geq U_{\varepsilon}^{n}(x) \geq 0 \\
(1+C h) \exp (\theta f(\bar{u}) T) \bar{v} & \geq V_{\varepsilon}^{n}(x) \geq 0
\end{aligned}
$$

for all $x \in \overline{\Omega^{h}}$. In addition, we have that

$$
\left(\theta U_{\varepsilon}^{n}+V_{\varepsilon}^{n}, 1\right)^{h}=\left(\theta U_{\varepsilon}^{0}+V_{\varepsilon}^{0}, 1\right)^{h} .
$$

Proof. This proof is almost identical to proofs of Theorem 2.1 and Lemma 2.4 in Barrett and Nürnberg. ${ }^{1}$ We just highlight the key difference, which is $(3.4 \mathrm{~b})$ whose structure is simpler to that of its analogue in Barrett and Nürnberg. ${ }^{1}$ The monotonicity of $\psi_{\varepsilon}$ yields the existence and uniqueness of $W_{\varepsilon}^{n}$, and hence $V_{\varepsilon}^{n}$. Moreover, (3.6) ensures the $\mathcal{M}$-matrix structure of the diffusion term, which leads to the required inverse isotone homeomorphism structure, see e.g. §13.5.6 in Ortega and Rheinboldt, ${ }^{9}$ of (3.4b). Finally, the bound on $\mathcal{V}_{M}^{0}$ used in (3.21) follows immediately from (3.3), (3.1) and (3.9).

Remark 3.2. If $v^{0} \in W^{1, p}(\Omega)$ with $p>d$, then it is more convenient to choose $V_{\varepsilon}^{0} \equiv I^{h} v^{0}$. It is easily shown that all the results of this paper still hold; and in particular, the factor $(1+C h)$ in $(3.21)$ can be removed.

Lemma 3.1. Under the assumptions of Theorem 3.1, we have that

$$
\begin{aligned}
& \max _{n=1 \rightarrow N} c\left\|U_{\varepsilon}^{n}\right\|_{1, \Omega^{h}}^{2}+\sum_{n=1}^{N} \tau_{n}\left\|d_{t} U_{\varepsilon}^{n}\right\|_{0, \Omega^{h}}^{2}+c \sum_{n=1}^{N}\left|U_{\varepsilon}^{n}-U_{\varepsilon}^{n-1}\right|_{1, \Omega^{h}}^{2} \leq C, \\
& \sum_{n=1}^{N} \tau_{n}\left\|\left[I^{h}\left[b\left(U_{\varepsilon}^{n}\right)\right]\right]^{\frac{1}{2}} \nabla W_{\varepsilon}^{n}\right\|_{0, \Omega^{h}}^{2} \leq C .
\end{aligned}
$$

Proof. The bound (3.23a) follows immediately from choosing $\chi \equiv U_{\varepsilon}^{n}-U_{\varepsilon}^{n-1}$ in (3.4a), summing over $n=1 \rightarrow k$, for an integer $k \in[1, N]$, and noting (3.18), 
(3.21), (3.10a), (3.11), (1.5) and (3.5c). The bound (3.23b) follows immediately from choosing $\chi \equiv \tau_{n} W_{\varepsilon}^{n}$ in (3.4b), summing over $n=1 \rightarrow N$, and noting (3.19) and (3.21).

\subsection{Error Analysis}

Let $u_{\varepsilon}^{n}(\cdot):=u_{\varepsilon}\left(\cdot, t_{n}\right)$ for $n \geq 0$, and $\widetilde{u}_{\varepsilon}^{0}:=u^{0}$,

$$
\widetilde{u}_{\varepsilon}^{n}(\cdot):=\frac{1}{\tau_{n}} \int_{t_{n-1}}^{t_{n}} u_{\varepsilon}(\cdot, t) d t \quad \text { for } n \geq 1 \quad \text { and } \quad e_{u}^{n}:=\widetilde{u}_{\varepsilon}^{n}-U_{\varepsilon}^{n} \quad \text { for } n \geq 0,
$$

with analogous notation for $v_{\varepsilon}$ and $w_{\varepsilon}:=\psi_{\varepsilon}\left(v_{\varepsilon}\right)$. Then for example, we have for $n \geq 1$ and any $\widehat{t} \in\left[t_{n-1}, t_{n}\right]$ that

$$
\left|\widetilde{u}_{\varepsilon}^{n}(\cdot)-u_{\varepsilon}(\cdot, \widehat{t})\right|^{2} \equiv \frac{1}{\tau_{n}^{2}}\left|\int_{t_{n-1}}^{t_{n}}\left[u_{\varepsilon}(\cdot, t)-u_{\varepsilon}(\cdot, \widehat{t})\right] d t\right|^{2} \leq \tau_{n} \int_{t_{n-1}}^{t_{n}}\left|\frac{\partial u_{\varepsilon}}{\partial t}(\cdot, t)\right|^{2} d t .
$$

Below we will need the summation by parts formula

$$
\sum_{n=1}^{k} r^{n}\left[s^{n}-s^{n-1}\right]=r^{k} s^{k}-r^{0} s^{0}-\sum_{n=1}^{k} s^{n-1}\left[r^{n}-r^{n-1}\right] .
$$

In addition, we will need the discrete Gronwall inequality:

$$
\begin{aligned}
\left(r^{0}\right)^{2}+\left(s^{0}\right)^{2} & \leq\left(q^{0}\right)^{2}, \\
\left(r^{k}\right)^{2}+\left(s^{k}\right)^{2} & \leq \sum_{n=0}^{k-1}\left(\zeta^{n}\right)^{2}\left(r^{n}\right)^{2}+\sum_{n=0}^{k}\left(q^{n}\right)^{2} \quad k \geq 1 \\
\Rightarrow \quad\left(r^{k}\right)^{2}+\left(s^{k}\right)^{2} & \leq \exp \left(\sum_{n=0}^{k-1}\left(\zeta^{n}\right)^{2}\right) \sum_{n=0}^{k}\left(q^{n}\right)^{2} \quad k \geq 1 .
\end{aligned}
$$

Finally, in order to analyse the error in our approximation we need to extend $U_{\varepsilon}^{n}$ and $V_{\varepsilon}^{n}$ from $\Omega^{h}$ to $\widetilde{\Omega}^{h}$, as described in (3.2). Therefore, in place of (3.21), we have for $n=1 \rightarrow N$ that

$$
\left|U_{\varepsilon}^{n}(x)\right| \leq 2 \bar{u} \quad \text { and } \quad\left|V_{\varepsilon}^{n}(x)\right| \leq 2(1+C h) \exp (\theta f(\bar{u}) T) \bar{v} \quad \forall x \in{\widetilde{\Omega^{h}}}^{h} .
$$

In particular, $U_{\varepsilon}^{n}$ and $V_{\varepsilon}^{n}$ may be negative on $\widetilde{\Omega}^{h}$, so that we have to extend $f, b$ and $\psi_{\varepsilon}$ for this analysis. Clearly, $f$ can be extended by zero, and $\psi_{\varepsilon}$ can be extended linearly so that $f \in C_{\text {loc }}^{0,1}(\mathbb{R})$ with $f$ increasing, and $\psi_{\varepsilon} \in C^{1}(\mathbb{R})$ convex satisfying $\psi_{\varepsilon}^{\prime}(s) \geq \psi_{\varepsilon}^{\prime}(0)$ for all $s \in \mathbb{R}$. If $b(0)=0$, we extend $b$ by zero; and (1.2) implies that $b^{\prime}(0)=0$ and hence $b \in C_{\text {loc }}^{1,1}(\mathbb{R})$. If $b(0)>0$, then one can choose a linear extension for $b^{\prime}(0) \leq 0$; and if $b^{\prime}(0)>0$ one can set $b(s)=\frac{1}{2} b(0)\left[1+e^{\frac{2 b^{\prime}(0) s}{b(0)}}\right]$ for $s \leq 0$. So in all cases, we obtain a non-negative $b \in C_{\text {loc }}^{1,1}(\mathbb{R})$ satisfying (1.2) for all $|r| \leq 2 \bar{u}$. 
Theorem 3.2. Let the assumptions of Corollary 2.2 hold, and the assumptions (A). Then for all $\varepsilon \leq \varepsilon_{1}, h \leq h_{0}$ and $\tau \leq \tau_{0}$, where $\tau_{0}$ is independent of $h$, we have the following error bound

$$
\begin{gathered}
\max _{n=1 \rightarrow N}\left\|\widetilde{u}_{\varepsilon}^{n}-U_{\varepsilon}^{n}\right\|_{0}^{2}+\sum_{n=1}^{N} \tau_{n}\left|\widetilde{u}_{\varepsilon}^{n}-U_{\varepsilon}^{n}\right|_{1}^{2} d t+\max _{n=1 \rightarrow N}\left\|\mathcal{G}\left(\widetilde{v}_{\varepsilon}^{n}-V_{\varepsilon}^{n}\right)\right\|_{1}^{2} \\
+\sum_{n=1}^{N} \int_{t_{n-1}}^{t_{n}}\left(b\left(u_{\varepsilon}\right)\left[\psi_{\varepsilon}\left(v_{\varepsilon}\right)-\psi_{\varepsilon}\left(V_{\varepsilon}^{n}\right)\right], v_{\varepsilon}-V_{\varepsilon}^{n}\right) d t \\
\leq C\left[\tau+\left[\psi_{\varepsilon}^{\prime}(0)\right]^{-1} h^{2}+\left[\tau_{\min }\right]^{-1} h^{2}\right] .
\end{gathered}
$$

Proof. Choosing $\phi \equiv e_{u}^{n}$ in (2.17a), integrating in time over $\left(t_{n-1}, t_{n}\right)$, and then subtracting (3.4a) with $\chi \equiv \tau_{n} \mathcal{R}^{h} e_{u}^{n}$ yields, on noting (3.18), that

$$
\begin{aligned}
& \frac{1}{2}\left[\left\|e_{u}^{n}\right\|_{0}^{2}+\left\|e_{u}^{n}-e_{u}^{n-1}\right\|_{0}^{2}-\left\|e_{u}^{n-1}\right\|_{0}^{2}\right]+c \tau_{n}\left|e_{u}^{n}\right|_{1}^{2}+\tau_{n}\left(V_{\varepsilon}^{n-1}\left[f\left(\widetilde{u}_{\varepsilon}^{n}\right)-f\left(U_{\varepsilon}^{n}\right)\right], e_{u}^{n}\right) \\
& =\tau_{n}\left(d_{t}\left(\widetilde{u}_{\varepsilon}^{n}-u_{\varepsilon}^{n}\right), e_{u}^{n}\right)+\tau_{n}\left[\left(d_{t} U_{\varepsilon}^{n}, \mathcal{R}^{h} e_{u}^{n}\right)^{h}-\left(d_{t} U_{\varepsilon}^{n}, e_{u}^{n}\right)\right] \\
& +c \tau_{n}\left[\left(\nabla U_{\varepsilon}^{n}, \nabla\left[\mathcal{R}^{h} e_{u}^{n}\right]\right)^{h}-\left(\nabla U_{\varepsilon}^{n}, \nabla e_{u}^{n}\right)\right] \\
& +\left(\tau_{n} f\left(\widetilde{u}_{\varepsilon}^{n}\right) V_{\varepsilon}^{n-1}-\int_{t_{n-1}}^{t_{n}} f\left(u_{\varepsilon}\right) v_{\varepsilon} d t, e_{u}^{n}\right) \\
& +\tau_{n}\left[\left(f\left(U_{\varepsilon}^{n}\right) V_{\varepsilon}^{n-1}, \mathcal{R}^{h} e_{u}^{n}\right)^{h}-\left(f\left(U_{\varepsilon}^{n}\right) V_{\varepsilon}^{n-1}, e_{u}^{n}\right)\right] \\
& =: \sum_{i=1}^{5} \mathcal{U}_{i}^{n}
\end{aligned}
$$

We now sum (3.30) from $n=1$ to an integer $k \in[1, N]$ and bound the terms on the right-hand-side. Applying (3.26), and noting (3.24) and (3.25), we have for any $\gamma>0$ that

$$
\begin{aligned}
\sum_{n=1}^{k} \mathcal{U}_{1}^{n} & =\left(\widetilde{u}_{\varepsilon}^{k}-u_{\varepsilon}^{k}, e_{u}^{k}\right)-\sum_{n=2}^{k}\left(\widetilde{u}_{\varepsilon}^{n-1}-u_{\varepsilon}^{n-1}, e_{u}^{n}-e_{u}^{n-1}\right) \\
& \leq \gamma\left[\left\|e_{u}^{k}\right\|_{0}^{2}+\sum_{n=2}^{k}\left\|e_{u}^{n}-e_{u}^{n-1}\right\|_{0}^{2}\right]+C_{\gamma} \tau \int_{0}^{t_{k}}\left\|\frac{\partial u_{\varepsilon}}{\partial t}\right\|_{0}^{2} d t
\end{aligned}
$$

From (3.14c), (3.10b) and (3.10a) we have that

$$
\sum_{n=1}^{k} \mathcal{U}_{2}^{n} \leq \gamma \sum_{n=1}^{k} \tau_{n}\left\|e_{u}^{n}\right\|_{1}^{2}+C_{\gamma} h^{2} \sum_{n=1}^{k} \tau_{n}\left\|d_{t} U_{\varepsilon}^{n}\right\|_{0, \Omega^{h}}^{2} .
$$


From (3.9), (3.13), (3.14c) and (3.10a) we have that

$$
\begin{gathered}
\sum_{n=1}^{k} \mathcal{U}_{3}^{n}=c \sum_{n=1}^{k} \tau_{n}\left[\left(\nabla U_{\varepsilon}^{n}, \nabla\left[\mathcal{R}^{h} e_{u}^{n}\right]\right)^{h}-\left(\nabla U_{\varepsilon}^{n}, \nabla\left[\mathcal{R}^{h} e_{u}^{n}\right]\right)\right] \\
+c \sum_{n=1}^{k} \tau_{n}\left(U_{\varepsilon}^{n},\left(I-\mathcal{R}^{h}\right) e_{u}^{n}\right) \\
\leq \gamma \sum_{n=1}^{k} \tau_{n}\left\|e_{u}^{n}\right\|_{1}^{2}+C_{\gamma} h^{2} \sum_{n=1}^{k} \tau_{n}\left\|U_{\varepsilon}^{n}\right\|_{1, \Omega^{h}}^{2}
\end{gathered}
$$

Applying (2.24), (3.25) and (2.18d), we have that

$$
\begin{aligned}
\sum_{n=1}^{k} \mathcal{U}_{4}^{n} \leq \gamma & \sum_{n=1}^{k} \tau_{n}\left[\left\|f\left(\widetilde{u}_{\varepsilon}^{n}\right) e_{u}^{n}\right\|_{1}^{2}+\left\|e_{u}^{n}\right\|_{0}^{2}\right] \\
& +C_{\gamma} \sum_{n=1}^{k} \tau_{n}\left\|\mathcal{G}\left(e_{v}^{n-1}+\left(\widetilde{v}_{\varepsilon}^{n}-v_{\varepsilon}^{n-1}\right)+\left(v_{\varepsilon}^{n-1}-\widetilde{v}_{\varepsilon}^{n-1}\right)\right)\right\|_{1}^{2} \\
& +C_{\gamma} \sum_{n=1}^{k} \tau_{n}\left\|\frac{1}{\tau_{n}} \int_{t_{n-1}}^{t_{n}}\left[f\left(\widetilde{u}_{\varepsilon}^{n}\right)-f\left(u_{\varepsilon}\right)\right] v_{\varepsilon} d t\right\|_{0}^{2} \\
\leq \gamma & C \sum_{n=1}^{k}\left[\tau_{n}\left\|e_{u}^{n}\right\|_{1}^{2}+\left(\int_{t_{n-1}}^{t_{n}}\left|u_{\varepsilon}\right|_{1, \infty}^{2} d t\right)\left\|e_{u}^{n}\right\|_{0}^{2}\right] \\
& +C_{\gamma}\left[\sum_{n=1}^{k} \tau_{n}\left\|\mathcal{G} e_{v}^{n-1}\right\|_{1}^{2}+\tau^{2} \int_{0}^{t_{k}}\left[\left\|\frac{\partial u_{\varepsilon}}{\partial t}\right\|_{0}^{2}+\left\|\mathcal{G} \frac{\partial v_{\varepsilon}}{\partial t}\right\|_{1}^{2}\right] d t\right]
\end{aligned}
$$

In order to analyse the next term, we first note that

$$
\begin{aligned}
\mathcal{U}_{5}^{n}= & \tau_{n}\left[\left(I^{h}\left[f\left(U_{\epsilon}^{n}\right) \mathcal{R}^{h} e_{u}^{n}\right], V_{\epsilon}^{n-1}\right)^{h}-\left(I^{h}\left[f\left(U_{\epsilon}^{n}\right) \mathcal{R}^{h} e_{u}^{n}\right], V_{\epsilon}^{n-1}\right)\right] \\
& \quad+\tau_{n}\left(\left(I^{h}-I\right)\left[f\left(U_{\epsilon}^{n}\right) \mathcal{R}^{h} e_{u}^{n}\right], V_{\epsilon}^{n-1}\right)+\tau_{n}\left(f\left(U_{\epsilon}^{n}\right) V_{\epsilon}^{n-1},\left(\mathcal{R}^{h}-I\right) e_{u}^{n}\right) \\
=: & I+I I+I I I .
\end{aligned}
$$

It follows from (3.10b) with $p=1,(3.28)$ and (3.10a) that

$$
\begin{aligned}
|I| & \leq C \tau_{n} h\left\|I^{h}\left[f\left(U_{\epsilon}^{n}\right) \mathcal{R}^{h} e_{u}^{n}\right]\right\|_{1,1, \Omega^{h}} \leq C \tau_{n} h \sum_{\kappa}|\kappa|\left\|I^{h}\left[f\left(U_{\epsilon}^{n}\right) \mathcal{R}^{h} e_{u}^{n}\right]\right\|_{1, \infty, \kappa} \\
& \leq C \tau_{n} h \sum_{\kappa}|\kappa|\left(\left|\mathcal{R}^{h} e_{u}^{n}\right|_{0, \infty, \kappa}+\left|\nabla U_{\epsilon}^{n}\right|_{0, \infty, \kappa}\left|\mathcal{R}^{h} e_{u}^{n}\right|_{0, \infty, \kappa}+\left|\nabla \mathcal{R}^{h} e_{u}^{n}\right|_{0, \infty, \kappa}\right) \\
& \leq C \tau_{n} h\left(1+\left|U_{\varepsilon}^{n}\right|_{1, \Omega^{h}}\right)\left\|\mathcal{R}^{h} e_{u}^{n}\right\|_{1, \Omega^{h}}
\end{aligned}
$$

Similarly, we obtain that

$$
|I I| \leq C \tau_{n} \sum_{\kappa} h_{\kappa}|\kappa|\left|\nabla\left[f\left(U_{\epsilon}^{n}\right) \mathcal{R}^{h} e_{u}^{n}\right]\right|_{0, \infty, \kappa} \leq C \tau_{n} h\left\|U_{\varepsilon}^{n}\right\|_{1, \Omega^{h}}\left\|\mathcal{R}^{h} e_{u}^{n}\right\|_{1, \Omega^{h}}
$$


Combining (3.35)-(3.37), and noting (3.28), (3.10a) and (3.14c) yields that

$$
\sum_{n=1}^{k} \mathcal{U}_{5}^{n} \leq \gamma \sum_{n=1}^{k} \tau_{n}\left\|e_{u}^{n}\right\|_{1}^{2}+C_{\gamma} h^{2}\left(1+\sum_{n=1}^{k} \tau_{n}\left\|U_{\varepsilon}^{n}\right\|_{1, \Omega^{h}}^{2}\right)
$$

Combining (3.30) with (3.31)-(3.34) and (3.38), and taking into account (2.18a), $(2.18 \mathrm{c})$ and (3.23a) we obtain after choosing $\gamma$ sufficiently small that for any integer $k \in[1, N]$

$$
\begin{gathered}
\left\|e_{u}^{k}\right\|_{0}^{2}+\sum_{n=1}^{k} \tau_{n}\left|e_{u}^{n}\right|_{1}^{2} \leq C \sum_{n=1}^{k}\left(\int_{t_{n-1}}^{t_{n}}\left(1+\left|u_{\varepsilon}\right|_{1, \infty}^{2}\right) d t\right)\left(\left\|e_{u}^{n}\right\|_{0}^{2}+\left\|\mathcal{G} e_{v}^{n}\right\|_{1}^{2}\right) \\
+\left\|e_{u}^{0}\right\|_{0}^{2}+\tau\left\|\mathcal{G} e_{v}^{0}\right\|_{1}^{2}+C\left(\tau+h^{2}\right) .
\end{gathered}
$$

Choosing $\phi \equiv \mathcal{G} e_{v}^{n}$ in (2.17b), integrating in time over $\left(t_{n-1}, t_{n}\right)$, and then subtracting (3.4b) with $\chi \equiv \tau_{n} \mathcal{G}^{h} e_{v}^{n}$ yields on noting (3.18) and (2.24) that

$$
\begin{aligned}
\frac{1}{2}\left[\left\|\mathcal{G} e_{v}^{n}\right\|_{1}^{2}+\right. & \left.\left\|\mathcal{G}\left(e_{v}^{n}-e_{v}^{n-1}\right)\right\|_{1}^{2}-\left\|\mathcal{G} e_{v}^{n-1}\right\|_{1}^{2}\right] \\
& \quad+\int_{t_{n-1}}^{t_{n}}\left(b\left(u_{\varepsilon}\right)\left[\psi_{\varepsilon}\left(v_{\varepsilon}\right)-\psi_{\varepsilon}\left(V_{\varepsilon}^{n}\right)\right], v_{\varepsilon}-V_{\varepsilon}^{n}\right) d t \\
= & \tau_{n}\left(d_{t}\left(\widetilde{v}_{\varepsilon}^{n}-v_{\varepsilon}^{n}\right), \mathcal{G} e_{v}^{n}\right)+\tau_{n}\left[\left(d_{t} V_{\varepsilon}^{n}, \mathcal{G}^{h} e_{v}^{n}\right)^{h}-\left(d_{t} V_{\varepsilon}^{n}, \mathcal{G} e_{v}^{n}\right)\right] \\
& +\left[\int_{t_{n-1}}^{t_{n}}\left(b\left(u_{\varepsilon}\right)\left[\psi_{\varepsilon}\left(v_{\varepsilon}\right)-\psi_{\varepsilon}\left(V_{\varepsilon}^{n}\right)\right], v_{\varepsilon}-V_{\varepsilon}^{n}\right) d t\right. \\
& \left.+\tau_{n}\left(b\left(U_{\varepsilon}^{n}\right) \nabla W_{\varepsilon}^{n}, \nabla\left[\mathcal{G}^{h} e_{v}^{n}\right]\right)^{h}-\left(\int_{t_{n-1}}^{t_{n}} b\left(u_{\varepsilon}\right) \nabla w_{\varepsilon} d t, \nabla\left[\mathcal{G} e_{v}^{n}\right]\right)\right] \\
& +\theta\left(\int_{t_{n-1}}^{t_{n}} f\left(u_{\varepsilon}\right) v_{\varepsilon} d t-\tau_{n} f\left(\widetilde{u}_{\varepsilon}^{n}\right) V_{\varepsilon}^{n-1}, \mathcal{G} e_{v}^{n}\right) \\
& +\tau_{n} \theta\left[\left(f\left(U_{\varepsilon}^{n}\right) V_{\varepsilon}^{n-1}, \mathcal{G} e_{v}^{n}\right)-\left(f\left(U_{\varepsilon}^{n}\right) V_{\varepsilon}^{n-1}, \mathcal{G}^{h} e_{v}^{n}\right)^{h}\right] \\
& +\tau_{n} \theta\left(V_{\varepsilon}^{n-1}\left[f\left(\widetilde{u}_{\varepsilon}^{n}\right)-f\left(U_{\varepsilon}^{n}\right)\right], \mathcal{G} e_{v}^{n}\right)=: \sum_{i=1}^{6} \mathcal{V}_{i}^{n} .
\end{aligned}
$$

We now sum (3.40) from $n=1$ to an integer $k \in[1, N]$ and bound the terms on the right-hand-side. Similarly to (3.31), we have, on noting in addition (2.24), that for any $\gamma>0$

$$
\sum_{n=1}^{k} \mathcal{V}_{1}^{n} \leq \gamma\left[\left\|\mathcal{G} e_{v}^{k}\right\|_{1}^{2}+\sum_{n=2}^{k}\left\|\mathcal{G}\left(e_{v}^{n}-e_{v}^{n-1}\right)\right\|_{1}^{2}\right]+C_{\gamma} \tau \int_{0}^{t_{k}}\left\|\mathcal{G} \frac{\partial v_{\varepsilon}}{\partial t}\right\|_{1}^{2} d t
$$


From (3.26), (3.14a), (3.10a), (3.10b) and (3.28) we have for any $\gamma>0$ that

$$
\begin{aligned}
\sum_{n=1}^{k} \mathcal{V}_{2}^{n}=[ & \left.\left(V_{\varepsilon}^{k}, \mathcal{G}^{h} e_{v}^{k}\right)^{h}-\left(V_{\varepsilon}^{k}, \mathcal{G} e_{v}^{k}\right)\right]-\left[\left(V_{\varepsilon}^{0}, \mathcal{G}^{h} e_{v}^{0}\right)^{h}-\left(V_{\varepsilon}^{0}, \mathcal{G} e_{v}^{0}\right)\right] \\
& -\sum_{n=1}^{k}\left[\left(V_{\varepsilon}^{n-1}, \mathcal{G}^{h}\left(e_{v}^{n}-e_{v}^{n-1}\right)\right)^{h}-\left(V_{\varepsilon}^{n-1}, \mathcal{G}\left(e_{v}^{n}-e_{v}^{n-1}\right)\right)\right] \\
\leq \gamma & {\left[\left\|\mathcal{G} e_{v}^{k}\right\|_{1}^{2}+\left\|\mathcal{G} e_{v}^{0}\right\|_{1}^{2}+\sum_{n=1}^{k}\left\|\mathcal{G}\left(e_{v}^{n}-e_{v}^{n-1}\right)\right\|_{1}^{2}\right]+C_{\gamma} \frac{h^{2}}{\tau_{\min }} . }
\end{aligned}
$$

As $\sum_{n=1}^{k} \mathcal{V}_{3}^{n}$ is the most difficult term to bound, we will leave this to the end. We now consider $\sum_{n=1}^{k} \mathcal{V}_{4}^{n}$. Similarly to (3.34), it follows from (2.24), (3.25), (2.18a), (2.18c) and (2.18d) that

$$
\begin{aligned}
\sum_{n=1}^{k} \mathcal{V}_{4}^{n} \leq & C \sum_{n=1}^{k} \tau_{n}\left[\left\|f\left(\widetilde{u}_{\varepsilon}^{n}\right) \mathcal{G} e_{v}^{n}\right\|_{1}^{2}+\left\|\mathcal{G} e_{v}^{n}\right\|_{0}^{2}\right] \\
& +C\left[\sum_{n=1}^{k} \tau_{n}\left\|\mathcal{G} e_{v}^{n-1}\right\|_{1}^{2}+\tau^{2} \int_{0}^{t_{k}}\left[\left\|\frac{\partial u_{\varepsilon}}{\partial t}\right\|_{0}^{2}+\left\|\mathcal{G} \frac{\partial v_{\varepsilon}}{\partial t}\right\|_{1}^{2}\right] d t\right] \\
\leq & C \sum_{n=1}^{k}\left(\int_{t_{n-1}}^{t_{n}}\left[1+\left|u_{\varepsilon}\right|_{1, \infty}^{2}\right] d t\right)\left\|\mathcal{G} e_{v}^{n}\right\|_{1}^{2}+\left\|\mathcal{G} e_{v}^{0}\right\|_{1}^{2}+C \tau^{2} .
\end{aligned}
$$

Similarly to (3.38), we have from (3.10b), (3.10a), (3.28), (3.36), (3.14a) and (3.23a) that

$$
\sum_{n=1}^{k} \mathcal{V}_{5}^{n} \leq C \sum_{n=1}^{k} \tau_{n}\left\|\mathcal{G} e_{v}^{n}\right\|_{1}^{2}+C h^{2}
$$

Next, on noting (3.28), we have that

$$
\sum_{n=1}^{k} \mathcal{V}_{6}^{n} \leq C \sum_{n=1}^{k} \tau_{n}\left[\left\|e_{u}^{n}\right\|_{0}^{2}+\left\|\mathcal{G} e_{v}^{n}\right\|_{0}^{2}\right]
$$

We now work on the final, and most difficult, term $\sum_{n=1}^{k} \mathcal{V}_{3}^{n}$. First, we rewrite

$$
\begin{gathered}
\mathcal{V}_{3}^{n}=\int_{t_{n-1}}^{t_{n}}\left(b\left(u_{\varepsilon}\right)\left[\psi_{\varepsilon}\left(v_{\varepsilon}\right)-\psi_{\varepsilon}\left(V_{\varepsilon}^{n}\right)\right], v_{\varepsilon}-V_{\varepsilon}^{n}\right) d t-\int_{t_{n-1}}^{t_{n}}\left(b\left(u_{\varepsilon}\right) \nabla w_{\varepsilon}, \nabla\left[\mathcal{G} e_{v}^{n}\right]\right) d t \\
+\tau_{n}\left(b\left(U_{\varepsilon}^{n}\right) \nabla W_{\varepsilon}^{n}, \nabla\left[\mathcal{G}^{h} e_{v}^{n}\right]\right)^{h}=: \sum_{i=1}^{3}\left(\mathcal{V}_{3}^{n}\right)_{i} .
\end{gathered}
$$

Noting (2.24), we have that

$$
\begin{aligned}
\left(\mathcal{V}_{3}^{n}\right)_{2} & =\int_{t_{n-1}}^{t_{n}}\left(w_{\varepsilon} \nabla\left[b\left(u_{\varepsilon}\right)\right]-\nabla\left[b\left(u_{\varepsilon}\right) w_{\varepsilon}\right], \nabla\left[\mathcal{G} e_{v}^{n}\right]\right) d t \\
& =\int_{t_{n-1}}^{t_{n}}\left[\left(w_{\varepsilon} \nabla\left[b\left(u_{\varepsilon}\right)\right], \nabla\left[\mathcal{G} e_{v}^{n}\right]\right)-\left(b\left(u_{\varepsilon}\right) w_{\varepsilon},(I-\mathcal{G}) e_{v}^{n}\right)\right] d t
\end{aligned}
$$


Similarly, on noting (3.15) and (3.12), we have that

$$
\begin{aligned}
\left(\mathcal{V}_{3}^{n}\right)_{3}= & \tau_{n}\left(\left[b\left(U_{\varepsilon}^{n}\right) \mathcal{I}-D\left(I^{h}\left[b\left(U_{\varepsilon}^{n}\right)\right]\right)\right] \nabla W_{\varepsilon}^{n}, \nabla\left[\mathcal{G}^{h} e_{v}^{n}\right]\right)^{h} \\
& +\tau_{n}\left[\left(D\left(I^{h}\left[b\left(U_{\varepsilon}^{n}\right)\right]\right) \nabla W_{\varepsilon}^{n}, \nabla\left[\mathcal{G}^{h} e_{v}^{n}\right]\right)^{h}-\left(D\left(I^{h}\left[b\left(U_{\varepsilon}^{n}\right)\right]\right) \nabla W_{\varepsilon}^{n}, \nabla\left[\mathcal{G}^{h} e_{v}^{n}\right]\right)\right] \\
& +\tau_{n}\left(\nabla\left(I^{h}\left[b\left(U_{\varepsilon}^{n}\right) W_{\varepsilon}^{n}\right]\right)-D\left(W_{\varepsilon}^{n}\right) \nabla I^{h}\left[b\left(U_{\varepsilon}^{n}\right)\right], \nabla\left[\mathcal{G}^{h} e_{v}^{n}\right]\right) \\
= & \tau_{n}\left(\left[b\left(U_{\varepsilon}^{n}\right) \mathcal{I}-D\left(I^{h}\left[b\left(U_{\varepsilon}^{n}\right)\right]\right)\right] \nabla W_{\varepsilon}^{n}, \nabla\left[\mathcal{G}^{h} e_{v}^{n}\right]\right)^{h} \\
& +\tau_{n}\left[\left(D\left(I^{h}\left[b\left(U_{\varepsilon}^{n}\right)\right]\right) \nabla W_{\varepsilon}^{n}, \nabla\left[\mathcal{G}^{h} e_{v}^{n}\right]\right)^{h}-\left(D\left(I^{h}\left[b\left(U_{\varepsilon}^{n}\right)\right]\right) \nabla W_{\varepsilon}^{n}, \nabla\left[\mathcal{G}^{h} e_{v}^{n}\right]\right)\right] \\
& +\tau_{n}\left(I^{h}\left[b\left(U_{\varepsilon}^{n}\right) W_{\varepsilon}^{n}\right],\left(I-\mathcal{G}^{h}\right) e_{v}^{n}\right)-\left(D\left(W_{\varepsilon}^{n}\right) \nabla I^{h}\left[b\left(U_{\varepsilon}^{n}\right)\right], \nabla\left[\mathcal{G}^{h} e_{v}^{n}\right]\right) .
\end{aligned}
$$

Combining (3.46), (3.47) and (3.48), we obtain that

$$
\begin{aligned}
\sum_{n=1}^{k} \mathcal{V}_{3}^{n}= & \sum_{n=1}^{k} \int_{t_{n-1}}^{t_{n}}\left(b\left(u_{\varepsilon}\right)\left[\psi_{\varepsilon}\left(v_{\varepsilon}\right)-\psi_{\varepsilon}\left(V_{\varepsilon}^{n}\right)\right], v_{\varepsilon}-\widetilde{v}_{\varepsilon}^{n}\right) d t \\
& +\sum_{n=1}^{k} \int_{t_{n-1}}^{t_{n}}\left(I^{h}\left[b\left(U_{\varepsilon}^{n}\right) W_{\varepsilon}^{n}\right]-b\left(u_{\varepsilon}\right) \psi_{\varepsilon}\left(V_{\varepsilon}^{n}\right),\left(I-\mathcal{G}^{h}\right) e_{v}^{n}\right) d t \\
& +\sum_{n=1}^{k} \int_{t_{n-1}}^{t_{n}}\left(w_{\varepsilon} \nabla\left[b\left(u_{\varepsilon}\right)\right]-D\left(W_{\varepsilon}^{n}\right) \nabla I^{h}\left[b\left(U_{\varepsilon}^{n}\right)\right], \nabla\left[\mathcal{G}^{h} e_{v}^{n}\right]\right) d t \\
& +\sum_{n=1}^{k} \tau_{n}\left(\left[b\left(U_{\varepsilon}^{n}\right) \mathcal{I}-D\left(I^{h}\left[b\left(U_{\varepsilon}^{n}\right)\right]\right)\right] \nabla W_{\varepsilon}^{n}, \nabla\left[\mathcal{G}^{h} e_{v}^{n}\right]\right)^{h} \\
& +\sum_{n=1}^{k} \tau_{n}\left[\left(D\left(I^{h}\left[b\left(U_{\varepsilon}^{n}\right)\right]\right) \nabla W_{\varepsilon}^{n}, \nabla\left[\mathcal{G}^{h} e_{v}^{n}\right]\right)^{h}-\left(D\left(I^{h}\left[b\left(U_{\varepsilon}^{n}\right)\right]\right) \nabla W_{\varepsilon}^{n}, \nabla\left[\mathcal{G}^{h} e_{v}^{n}\right]\right)\right] \\
& +\sum_{n=1}^{k} \int_{t_{n-1}}^{t_{n}}\left(b\left(u_{\varepsilon}\right)\left[\psi_{\varepsilon}\left(v_{\varepsilon}\right)-\psi_{\varepsilon}\left(V_{\varepsilon}^{n}\right)\right], \mathcal{G} e_{v}^{n}\right) d t \\
& +\sum_{n=1}^{k} \int_{t_{n-1}}^{t_{n}}\left(\psi_{\varepsilon}\left(v_{\varepsilon}\right) \nabla\left[b\left(u_{\varepsilon}\right)\right], \nabla\left[\left(\mathcal{G}-\mathcal{G}^{h}\right) e_{v}^{n}\right]\right) d t \\
& +\sum_{n=1}^{k} \int_{t_{n-1}}^{t_{n}}\left(b\left(u_{\varepsilon}\right) \psi_{\varepsilon}\left(V_{\varepsilon}^{n}\right),\left(\mathcal{G}-\mathcal{G}^{h}\right) e_{v}^{n}\right) d t:=\sum_{i=1}^{8}\left(\mathcal{V}_{3}^{k}\right)_{i} .
\end{aligned}
$$


Noting (2.18a)-(2.18d), (3.28) and (3.25), we have that

$$
\begin{aligned}
\left(\mathcal{V}_{3}^{k}\right)_{1}= & \sum_{n=1}^{k} \int_{t_{n-1}}^{t_{n}}\left[\left(\left(b\left(u_{\varepsilon}\right)-b\left(\widetilde{u}_{\varepsilon}^{n}\right)\right)\left[\psi_{\varepsilon}\left(v_{\varepsilon}\right)-\psi_{\varepsilon}\left(V_{\varepsilon}^{n}\right)\right], v_{\varepsilon}-\widetilde{v}_{\varepsilon}^{n}\right)\right. \\
& \left.+\left(b\left(\widetilde{u}_{\varepsilon}^{n}\right) \psi_{\varepsilon}\left(v_{\varepsilon}\right), v_{\varepsilon}-\widetilde{v}_{\varepsilon}^{n}\right)\right] d t \\
\leq & C \sum_{n=1}^{k} \int_{t_{n-1}}^{t_{n}}\left[C\left\|u_{\varepsilon}-\widetilde{u}_{\varepsilon}^{n}\right\|_{0}+\left\|b\left(\widetilde{u}_{\varepsilon}^{n}\right) \psi_{\varepsilon}\left(v_{\varepsilon}\right)\right\|_{1}\left\|v_{\varepsilon}-\widetilde{v}_{\varepsilon}^{n}\right\|_{\left(H^{1}\right)^{\prime}}\right] d t \\
\leq & C \tau\left[\left(\int_{0}^{t_{k}}\left\|\frac{\partial u_{\varepsilon}}{\partial t}\right\|_{0}^{2} d t\right)^{\frac{1}{2}}+\sup _{t \in\left(0, t_{k}\right)}\left\|\frac{\partial v_{\varepsilon}}{\partial t}\right\|_{\left(H^{1}\right)^{\prime}}\right] \leq C \tau .
\end{aligned}
$$

In order to treat $\left(\mathcal{V}_{7}^{k}\right)_{2}$, we first note that

$$
\begin{aligned}
\left(I^{h}\right. & {\left.\left[b\left(U_{\varepsilon}^{n}\right) W_{\varepsilon}^{n}\right]-b\left(u_{\varepsilon}\right) \psi_{\varepsilon}\left(V_{\varepsilon}^{n}\right),\left(I-\mathcal{G}^{h}\right) e_{v}^{n}\right) } \\
= & \left(\left(I^{h}-I\right)\left[b\left(U_{\varepsilon}^{n}\right) W_{\varepsilon}^{n}\right],\left(I-\mathcal{G}^{h}\right) e_{v}^{n}\right)+\left(b\left(U_{\varepsilon}^{n}\right)\left[W_{\varepsilon}^{n}-\psi_{\varepsilon}\left(V_{\varepsilon}^{n}\right)\right],\left(I-\mathcal{G}^{h}\right) e_{v}^{n}\right) \\
& \quad+\left(\left[b\left(U_{\varepsilon}^{n}\right)-b\left(u_{\varepsilon}\right)\right] \psi_{\varepsilon}\left(V_{\varepsilon}^{n}\right), e_{v}^{n}\right)-\left(\left[b\left(U_{\varepsilon}^{n}\right)-b\left(u_{\varepsilon}\right)\right] \psi_{\varepsilon}\left(V_{\varepsilon}^{n}\right), \mathcal{G}^{h} e_{v}^{n}\right) \\
= & : I+I I+I I I+I V .
\end{aligned}
$$

Similarly to (3.36), on noting (3.5b), (3.28) and (2.18d), we have that

$$
|I| \leq C \sum_{\kappa \in \mathcal{T}^{h}} h_{\kappa}^{2}\left[|\kappa|\left|b\left(U_{\varepsilon}^{n}\right) W_{\varepsilon}^{n}\right|_{2, \infty, \kappa}+\left|b\left(U_{\varepsilon}^{n}\right) W_{\varepsilon}^{n}\right|_{2, \kappa}\left\|\mathcal{G}^{h} e_{v}^{n}\right\|_{0, \kappa}\right] .
$$

Clearly, we have that

$$
\left|\frac{\partial^{2}}{\partial x_{i} \partial x_{j}}\left[b\left(U_{\varepsilon}^{n}\right) W_{\varepsilon}^{n}\right]\right| \leq C\left|\nabla U_{\varepsilon}^{n}\right|^{2}\left|W_{\varepsilon}^{n}\right|+\left|b^{\prime}\left(U_{\varepsilon}^{n}\right)\right|\left|\nabla U_{\varepsilon}^{n}\right|\left|\nabla W_{\varepsilon}^{n}\right| ;
$$

so that on noting (3.28), (3.5a) and (1.2) we have that

$$
h_{\kappa}\left|b\left(U_{\varepsilon}^{n}\right) W_{\varepsilon}^{n}\right|_{2, \kappa} \leq C\left|U_{\varepsilon}^{n}\right|_{1, \kappa}+C\left(\int_{\kappa} b\left(U_{\varepsilon}^{n}\right)\left|\nabla W_{\varepsilon}^{n}\right|^{2}\right)^{\frac{1}{2}} .
$$

Furthermore, since for any $x \in \kappa$

$$
\left|b^{\prime}\left(U_{\varepsilon}^{n}\right)\right|_{0, \infty, \kappa} \leq\left|\left[b^{\prime}\left(U_{\varepsilon}^{n}\right)\right](x)\right|+C h_{\kappa}\left|U_{\varepsilon}^{n}\right|_{1, \infty, \kappa} ;
$$

we have, on noting (3.5a) and (1.2), that

$$
|\kappa|\left|b\left(U_{\varepsilon}^{n}\right) W_{\varepsilon}^{n}\right|_{2, \infty, \kappa} \leq C\left|U_{\varepsilon}^{n}\right|_{1, \kappa}^{2}+C \int_{\kappa} b\left(U_{\varepsilon}^{n}\right)\left|\nabla W_{\varepsilon}^{n}\right|^{2} .
$$

Hence in conclusion, on noting (3.23a) and (3.10a), we have that

$$
|I| \leq C h^{2}+C h^{2} \int_{\Omega^{h}} b\left(U_{\varepsilon}^{n}\right)\left|\nabla W_{\varepsilon}^{n}\right|^{2}+C\left\|\mathcal{G}^{h} e_{v}^{n}\right\|_{0}^{2} .
$$

Noting (3.5b) and (3.28), we have that

$$
\begin{aligned}
\left\|\left(I-I^{h}\right)\left[b\left(U_{\varepsilon}^{n}\right)\right]\right\|_{0,1, \kappa} & \leq|\kappa|\left\|\left(I-I^{h}\right)\left[b\left(U_{\varepsilon}^{n}\right)\right]\right\|_{0, \infty, \kappa} \\
& \leq C|\kappa| h_{\kappa}^{2}\left|\nabla U_{\varepsilon}^{n}\right|_{0, \infty, \kappa}^{2} \leq C h_{\kappa}^{2}\left|U_{\varepsilon}^{n}\right|_{1, \kappa}^{2} .
\end{aligned}
$$


Therefore (3.58), (3.5a) and (3.28) yield that

$$
\int_{\kappa} b\left(U_{\varepsilon}^{n}\right)\left|\nabla W_{\varepsilon}^{n}\right|^{2} \leq \int_{\kappa} I^{h}\left[b\left(U_{\varepsilon}^{n}\right)\right]\left|\nabla W_{\varepsilon}^{n}\right|^{2}+C \int_{\kappa}\left|\nabla U_{\varepsilon}^{n}\right|^{2} .
$$

Inserting (3.59) into (3.57), and noting (3.23a), we infer that

$$
|I| \leq C h^{2}+C h^{2} \int_{\Omega^{h}} I^{h}\left[b\left(U_{\varepsilon}^{n}\right)\right]\left|\nabla W_{\varepsilon}^{n}\right|^{2}+C\left\|\mathcal{G}^{h} e_{v}^{n}\right\|_{0}^{2} .
$$

Next we consider $I I$. Noting that (1.2) implies that $\sqrt{b}(\cdot)$ is locally Lipschitz continuous, and (3.8), (2.18d) and (3.28), we have that

$$
\begin{aligned}
& \left|\int_{\Omega} b\left(U_{\varepsilon}^{n}\right)\left(I^{h}\left[\psi_{\varepsilon}\left(V_{\varepsilon}^{n}\right)\right]-\psi_{\varepsilon}\left(V_{\varepsilon}^{n}\right)\right)\left(I-\mathcal{G}^{h}\right) e_{v}^{n}\right| \\
& \leq C h \int_{\Omega} b\left(U_{\varepsilon}^{n}\right)\left|\nabla W_{\varepsilon}^{n}\right|\left[\left|e_{v}^{n}\right|+\left|\mathcal{G}^{h} e_{v}^{n}\right|\right] \\
& \leq C h \int_{\Omega}\left(\left|\sqrt{b\left(U_{\varepsilon}^{n}\right)}-\sqrt{b\left(u_{\varepsilon}\right)}\right|+\sqrt{b\left(u_{\varepsilon}\right)}\right) \sqrt{b\left(U_{\varepsilon}^{n}\right)}\left|\nabla W_{\varepsilon}^{n}\right|\left[\left|e_{v}^{n}\right|+\left|\mathcal{G}^{h} e_{v}^{n}\right|\right] \\
& \quad \leq \gamma \psi_{\varepsilon}^{\prime}(0) \int_{\Omega} b\left(u_{\varepsilon}\right)\left[\left|v_{\varepsilon}-V_{\varepsilon}^{n}\right|^{2}+\left|v_{\varepsilon}-\widetilde{v}_{\varepsilon}^{n}\right|^{2}\right]+C\left[\left\|e_{u}^{n}\right\|_{0}^{2}+\left\|\mathcal{G}^{h} e_{v}^{n}\right\|_{0}^{2}\right] \\
& \quad+C\left\|u_{\varepsilon}-\widetilde{u}_{\varepsilon}^{n}\right\|_{0}^{2}+C_{\gamma}\left[\psi_{\varepsilon}^{\prime}(0)\right]^{-1} h^{2} \int_{\Omega} b\left(U_{\varepsilon}^{n}\right)\left|\nabla W_{\varepsilon}^{n}\right|^{2} .
\end{aligned}
$$

Hence the monotonicity of $\psi_{\varepsilon},(3.25)$ (3.59) for $\kappa \cap \Omega,(3.23 \mathrm{a})$ and (3.10a) yield that

$$
\begin{aligned}
|I I| \leq \gamma\left(b\left(u_{\varepsilon}\right)\right. & {\left.\left[\psi_{\varepsilon}\left(v_{\varepsilon}\right)-\psi_{\varepsilon}\left(V_{\varepsilon}^{n}\right)\right], v_{\varepsilon}-V_{\varepsilon}^{n}\right) } \\
& +C \tau_{n} \int_{t_{n-1}}^{t_{n}}\left[\psi_{\varepsilon}^{\prime}(0)\left\|\frac{\partial v_{\varepsilon}}{\partial t}\right\|_{0}^{2}+\left\|\frac{\partial u_{\varepsilon}}{\partial t}\right\|_{0}^{2}\right] d t+C\left\|\mathcal{G}^{h} e_{v}^{n}\right\|_{0}^{2} \\
& +C\left\|e_{u}^{n}\right\|_{0}^{2}+C_{\gamma}\left[\psi_{\varepsilon}^{\prime}(0)\right]^{-1} h^{2}\left(1+\int_{\Omega^{h}} I^{h}\left[b\left(U_{\varepsilon}^{n}\right)\right]\left|\nabla W_{\varepsilon}^{n}\right|^{2}\right) .
\end{aligned}
$$

Similarly to $T_{1}$ in (2.27), as bounded in (2.28), and (3.50), we have that

$$
\begin{aligned}
\int_{t_{n-1}}^{t_{n}} I I I d t=\int_{t_{n-1}}^{t_{n}}\left[\left(\left[b\left(U_{\varepsilon}^{n}\right)-b\left(u_{\varepsilon}\right)\right] \psi_{\varepsilon}\left(V_{\varepsilon}^{n}\right), v_{\varepsilon}-V_{\varepsilon}^{n}\right)\right. & \\
& \left.+\left(\left[b\left(\widetilde{u}_{\varepsilon}^{n}\right)-b\left(u_{\varepsilon}\right)\right] \psi_{\varepsilon}\left(V_{\varepsilon}^{n}\right), \widetilde{v}_{\varepsilon}^{n}-v_{\varepsilon}\right)\right] d t \\
\leq \gamma \int_{t_{n-1}}^{t_{n}}\left(b ( u _ { \varepsilon } ) \left[\psi_{\varepsilon}\left(v_{\varepsilon}\right)-\right.\right. & \left.\left.\psi_{\varepsilon}\left(V_{\varepsilon}^{n}\right)\right], v_{\varepsilon}-V_{\varepsilon}^{n}\right) d t+C_{\gamma} \tau_{n}\left\|e_{u}^{n}\right\|_{0}^{2} \\
& +C_{\gamma} \tau_{n}^{\frac{3}{2}}\left(\int_{t_{n-1}}^{t_{n}}\left\|\frac{\partial u_{\varepsilon}}{\partial t}\right\|_{0}^{2} d t\right)^{\frac{1}{2}} .
\end{aligned}
$$

Finally on noting (3.28) and (3.25), we have that

$$
|I V| \leq C\left[\left\|e_{u}^{n}\right\|_{0}^{2}+\left\|\mathcal{G}^{h} e_{v}^{n}\right\|_{0}^{2}+\tau_{n} \int_{t_{n-1}}^{t_{n}}\left\|\frac{\partial u_{\varepsilon}}{\partial t}\right\|_{0}^{2} d t\right] .
$$


Combining (3.51), (3.60) and (3.62)-(3.64), and applying the bounds (3.23b), (2.18a), (2.18c) and (3.14a), we obtain in conclusion that

$$
\begin{gathered}
\left(\mathcal{V}_{3}^{k}\right)_{2} \leq \gamma \sum_{n=1}^{k} \int_{t_{n-1}}^{t_{n}}\left(b\left(u_{\varepsilon}\right)\left[\psi_{\varepsilon}\left(v_{\varepsilon}\right)-\psi_{\varepsilon}\left(V_{\varepsilon}^{n}\right)\right], v_{\varepsilon}-V_{\varepsilon}^{n}\right) d t+C_{\gamma}\left[\psi_{\varepsilon}^{\prime}(0)\right]^{-1} h^{2} \\
+C \tau+C \sum_{n=1}^{k} \tau_{n}\left[\left\|e_{u}^{n}\right\|_{0}^{2}+\left\|\mathcal{G} e_{v}^{n}\right\|_{1}^{2}\right] .
\end{gathered}
$$

We now consider $\left(\mathcal{V}_{3}^{k}\right)_{3}$. First, we note that

$$
\left(\mathcal{V}_{3}^{k}\right)_{3} \leq \sum_{n=1}^{k} \int_{t_{n-1}}^{t_{n}}\left\|w_{\varepsilon} \nabla\left[b\left(u_{\varepsilon}\right)\right]-D\left(W_{\varepsilon}^{n}\right) \nabla I^{h}\left[b\left(U_{\varepsilon}^{n}\right)\right]\right\|_{0}\left\|\mathcal{G} e_{v}^{n}\right\|_{1} d t .
$$

Next we note from (3.16a)-(3.16b) and (3.28) that

$$
\begin{aligned}
\| w_{\varepsilon} \nabla\left[b\left(u_{\varepsilon}\right)\right] & -D\left(W_{\varepsilon}^{n}\right) \nabla I^{h}\left[b\left(U_{\varepsilon}^{n}\right)\right] \|_{0} \\
& \leq\left\|\left[w_{\varepsilon} \mathcal{I}-D\left(W_{\varepsilon}^{n}\right)\right] \nabla\left[b\left(u_{\varepsilon}\right)\right]\right\|_{0}+C\left|b\left(u_{\varepsilon}\right)-I^{h}\left[b\left(U_{\varepsilon}^{n}\right)\right]\right|_{1} .
\end{aligned}
$$

From (3.28) and (2.18d) we have that

$$
\begin{aligned}
\left|b\left(u_{\varepsilon}\right)-b\left(U_{\varepsilon}^{n}\right)\right|_{1} & \leq C\left\|\left[b^{\prime}\left(u_{\varepsilon}\right)-b^{\prime}\left(U_{\varepsilon}^{n}\right)\right] \nabla u_{\varepsilon}\right\|_{0}+C\left|u_{\varepsilon}-U_{\varepsilon}^{n}\right|_{1} \\
& \leq C\left|u_{\varepsilon}\right|_{1, \infty}\left\|u_{\varepsilon}-U_{\varepsilon}^{n}\right\|_{0}+C\left|u_{\varepsilon}-U_{\varepsilon}^{n}\right|_{1} .
\end{aligned}
$$

Similarly to (3.52) and (3.53), it follows from (3.5b), (3.5a), (3.28), (2.18d) and (3.10a) that for any $\kappa \in \mathcal{T}^{h}$

$$
\begin{aligned}
\left|\left(I-I^{h}\right)\left[b\left(U_{\varepsilon}^{n}\right)\right]\right|_{1, \kappa \cap \Omega} & \leq C|\kappa \cap \Omega|^{\frac{1}{2}}\left|\left(I-I^{h}\right)\left[b\left(U_{\varepsilon}^{n}\right)\right]\right|_{1, \infty, \kappa \cap \Omega} \leq C h_{\kappa}\left|U_{\varepsilon}^{n}\right|_{1,4, \kappa \cap \Omega}^{2} \\
& \leq C h_{\kappa}\left[\left|U_{\varepsilon}^{n}-I^{h} u_{\varepsilon}\right|_{1,4, \kappa \cap \Omega}^{2}+\left|I^{h} u_{\varepsilon}\right|_{1,4, \kappa \cap \Omega}^{2}\right] \\
& \leq C\left|U_{\varepsilon}^{n}-I^{h} u_{\varepsilon}\right|_{1, \kappa \cap \Omega}+C h_{\kappa}\left|I^{h} u_{\varepsilon}\right|_{1,4, \kappa \cap \Omega}^{2} .
\end{aligned}
$$

Hence on combining (3.68) and (3.69), we obtain, on noting (3.11), (2.18a), and that $W^{1,4}(\Omega) \hookrightarrow C(\bar{\Omega})$, as $d \leq 3$, and so $\left|I^{h} u_{\varepsilon}\right|_{1,4, \Omega} \leq C\left|u_{\varepsilon}\right|_{1,4, \Omega}$, that

$$
\begin{aligned}
\left|b\left(u_{\varepsilon}\right)-I^{h}\left[b\left(U_{\varepsilon}^{n}\right)\right]\right|_{1} \leq C\left[\left|u_{\varepsilon}\right|_{1, \infty}\left\|u_{\varepsilon}-U_{\varepsilon}^{n}\right\|_{0}+\left|u_{\varepsilon}-U_{\varepsilon}^{n}\right|_{1}\right] & \\
& +C h\left[\left|u_{\varepsilon}\right|_{2}+\left|u_{\varepsilon}\right|_{1, \infty}\left|u_{\varepsilon}\right|_{1}\right] .
\end{aligned}
$$

Next we note that

$$
\left\|\left[w_{\varepsilon} \mathcal{I}-D\left(W_{\varepsilon}^{n}\right)\right] \nabla\left[b\left(u_{\varepsilon}\right)\right]\right\|_{0} \leq C\left|u_{\varepsilon}\right|_{1, \infty}\left\|b^{\prime}\left(u_{\varepsilon}\right)\left[w_{\varepsilon} \mathcal{I}-D\left(W_{\varepsilon}^{n}\right)\right]\right\|_{0} ;
$$

and (1.2), (3.16a)-(3.16b), (2.18d) and (3.28) yield that

$$
\begin{aligned}
&\left\|b^{\prime}\left(u_{\varepsilon}\right)\left[w_{\varepsilon} \mathcal{I}-D\left(W_{\varepsilon}^{n}\right)\right]\right\|_{0} \\
& \leq\left\|b^{\prime}\left(u_{\varepsilon}\right)\left[\psi_{\varepsilon}\left(v_{\varepsilon}\right)-\psi_{\varepsilon}\left(V_{\varepsilon}^{n}\right)\right]\right\|_{0}+\left\|b^{\prime}\left(U_{\varepsilon}^{n}\right)\left[\psi_{\varepsilon}\left(V_{\varepsilon}^{n}\right) \mathcal{I}-D\left(W_{\varepsilon}^{n}\right)\right]\right\|_{0} \\
&+C\left\|b^{\prime}\left(u_{\varepsilon}\right)-b^{\prime}\left(U_{\varepsilon}^{n}\right)\right\|_{0} \\
& \leq C\left\|\left[b\left(u_{\varepsilon}\right)\right]^{\frac{1}{2}}\left[\psi_{\varepsilon}\left(v_{\varepsilon}\right)-\psi_{\varepsilon}\left(V_{\varepsilon}^{n}\right)\right]\right\|_{0}+C\left\|\left[b\left(U_{\varepsilon}^{n}\right)\right]^{\frac{1}{2}}\left[\psi_{\varepsilon}\left(V_{\varepsilon}^{n}\right) \mathcal{I}-D\left(W_{\varepsilon}^{n}\right)\right]\right\|_{0} \\
&+C\left\|u_{\varepsilon}-U_{\varepsilon}^{n}\right\|_{0}
\end{aligned}
$$


In addition, it follows from (3.8) and (3.17) that

$$
\left\|\left[b\left(U_{\varepsilon}^{n}\right)\right]^{\frac{1}{2}}\left[\psi_{\varepsilon}\left(V_{\varepsilon}^{n}\right) \mathcal{I}-D\left(W_{\varepsilon}^{n}\right)\right]\right\|_{0} \leq C h\left\|\left[b\left(U_{\varepsilon}^{n}\right)\right]^{\frac{1}{2}} \nabla W_{\varepsilon}^{n}\right\|_{0} .
$$

Therefore on combining (3.66), (3.67) and (3.70)-(3.73), and noting the monotonicity of $\psi_{\varepsilon},(3.59)$ for $\kappa \cap \Omega,(3.23 \mathrm{a})-(3.23 \mathrm{~b}),(3.25)$ and (2.18a) we obtain that

$$
\begin{aligned}
\left(\mathcal{V}_{3}^{k}\right)_{3} \leq & \gamma \sum_{n=1}^{k} \int_{t_{n-1}}^{t_{n}}\left[\left\|\left[b\left(u_{\varepsilon}\right)\right]^{\frac{1}{2}}\left[\psi_{\varepsilon}\left(v_{\varepsilon}\right)-\psi_{\varepsilon}\left(V_{\varepsilon}^{n}\right)\right]\right\|_{0}^{2}+\left\|u_{\varepsilon}-U_{\varepsilon}^{n}\right\|_{1}^{2}\right] d t \\
& +C h^{2}\left[\int_{0}^{t_{k}}\left\|u_{\varepsilon}\right\|_{2}^{2} d t+\sum_{n=1}^{k} \tau_{n}\left\|\left[b\left(U_{\varepsilon}^{n}\right)\right]^{\frac{1}{2}} \nabla W_{\varepsilon}^{n}\right\|_{0}^{2}\right] \\
& +C_{\gamma} \sum_{n=1}^{k} \int_{t_{n-1}}^{t_{n}}\left[1+\left|u_{\varepsilon}\right|_{1, \infty}^{2}\right]\left\|\mathcal{G} e_{v}^{n}\right\|_{1}^{2} d t \\
\leq & \gamma \sum_{n=1}^{k} \int_{t_{n-1}}^{t_{n}}\left[\left(b\left(u_{\varepsilon}\right)\left[\psi_{\varepsilon}\left(v_{\varepsilon}\right)-\psi_{\varepsilon}\left(V_{\varepsilon}^{n}\right)\right], v_{\varepsilon}-V_{\varepsilon}^{n}\right)+\left\|e_{u}^{n}\right\|_{1}^{2}\right] d t \\
& +C\left[h^{2}+\tau^{2}\right]+C_{\gamma} \sum_{n=1}^{k}\left(\int_{t_{n-1}}^{t_{n}}\left[1+\left|u_{\varepsilon}\right|_{1, \infty}^{2}\right] d t\right)\left\|\mathcal{G} e_{v}^{n}\right\|_{1}^{2}
\end{aligned}
$$

Next we consider $\left(\mathcal{V}_{3}^{k}\right)_{4}$. First it follows from (3.10a) and (3.14a) that

$$
\left(\mathcal{V}_{3}^{k}\right)_{4} \leq \sum_{n=1}^{k} \tau_{n}\left\|\left[I^{h}\left[b\left(U_{\varepsilon}^{n}\right)\right] \mathcal{I}-D\left(I^{h}\left[b\left(U_{\varepsilon}^{n}\right)\right]\right)\right] \nabla W_{\varepsilon}^{n}\right\|_{0, \Omega^{h}}\left\|\mathcal{G} e_{v}^{n}\right\|_{1} .
$$

We have, on noting (3.17) and (1.2), that for any $\kappa \in \mathcal{T}^{h}$

$$
\begin{aligned}
\|\left[I^{h}\right. & {\left.\left[b\left(U_{\varepsilon}^{n}\right)\right] \mathcal{I}-D\left(I^{h}\left[b\left(U_{\varepsilon}^{n}\right)\right]\right)\right] \nabla W_{\varepsilon}^{n} \|_{0, \kappa}^{2} } \\
& \leq C|\kappa| h_{\kappa}^{2}\left|I^{h}\left[b\left(U_{\varepsilon}^{n}\right)\right]\right|_{1, \infty, \kappa}^{2}\left|W_{\varepsilon}^{n}\right|_{1, \infty, \kappa}^{2} \leq C|\kappa| h_{\kappa}^{2}\left|b\left(U_{\varepsilon}^{n}\right)\right|_{1, \infty, \kappa}^{2}\left|W_{\varepsilon}^{n}\right|_{1, \infty, \kappa}^{2} \\
& \leq C|\kappa| h_{\kappa}^{2}\left|b\left(U_{\varepsilon}^{n}\right)\right|_{0, \infty, \kappa}\left|U_{\varepsilon}^{n}\right|_{1, \infty, \kappa}^{2}\left|W_{\varepsilon}^{n}\right|_{1, \infty, \kappa}^{2} \\
& \leq C|\kappa| h_{\kappa}^{2}\left|b\left(U_{\varepsilon}^{n}\right)\right|_{0, \infty, \kappa}\left[\left|I^{h}\left[\widetilde{u}_{\varepsilon}^{n}\right]\right|_{1, \infty, \kappa}^{2}+\left|I^{h}\left[\widetilde{u}_{\varepsilon}^{n}\right]-U_{\varepsilon}^{n}\right|_{1, \infty, \kappa}^{2}\right]\left|W_{\varepsilon}^{n}\right|_{1, \infty, \kappa}^{2} .
\end{aligned}
$$

Similarly to (3.55), we have for any $x \in \kappa$, on noting (3.28) and (1.2) that

$$
\left|b\left(U_{\varepsilon}^{n}\right)\right|_{0, \infty, \kappa} \leq\left[b\left(U_{\varepsilon}^{n}\right)\right](x)+C h_{\kappa}\left|\left[b\left(U_{\varepsilon}^{n}\right)\right]^{\frac{1}{2}}(x)\right|\left|U_{\varepsilon}^{n}\right|_{1, \infty, \kappa}+C h_{\kappa}^{2}\left|U_{\varepsilon}^{n}\right|_{1, \infty, \kappa}^{2} .
$$

Combining (3.76) and (3.77), on noting (3.5a) and (3.28), yields that

$$
\begin{aligned}
& \left\|\left[I^{h}\left[b\left(U_{\varepsilon}^{n}\right)\right] \mathcal{I}-D\left(I^{h}\left[b\left(U_{\varepsilon}^{n}\right)\right]\right)\right] \nabla W_{\varepsilon}^{n}\right\|_{0, \Omega^{h}}^{2} \\
& \quad \leq C h^{2}\left|\widetilde{u}_{\varepsilon}^{n}\right|_{1, \infty}^{2}\left(\left|U_{\varepsilon}^{n}\right|_{1, \Omega^{h}}^{2}+\int_{\Omega^{h}} b\left(U_{\varepsilon}^{n}\right)\left|\nabla W_{\varepsilon}^{n}\right|^{2}\right)+C\left|I^{h}\left[\widetilde{u}_{\varepsilon}^{n}\right]-U_{\varepsilon}^{n}\right|_{1, \Omega^{h}}^{2} .
\end{aligned}
$$


Inserting (3.78) into (3.75), we obtain, on noting (3.23a), (3.11), (2.18a), (3.59) and (3.23b), that

$$
\begin{aligned}
\left(\mathcal{V}_{3}^{k}\right)_{4} \leq & \gamma \sum_{n=1}^{k} \tau_{n}\left|e_{u}^{n}\right|_{1}^{2}+C_{\gamma} \sum_{n=1}^{k} \int_{t_{n-1}}^{t_{n}}\left[1+\left|u_{\varepsilon}\right|_{1, \infty}^{2}\right] d t\left\|\mathcal{G} e_{v}^{n}\right\|_{1}^{2} \\
& +C h^{2} \sum_{n=1}^{k} \tau_{n}\left(\left|U_{\varepsilon}^{n}\right|_{1}^{2}+\int_{\Omega^{h}} b\left(U_{\varepsilon}^{n}\right)\left|\nabla W_{\varepsilon}^{n}\right|^{2}\right)+C \int_{0}^{t_{k}}\left|\left(I-I^{h}\right) u_{\varepsilon}\right|_{1}^{2} d t \\
\leq & \gamma \sum_{n=1}^{k} \tau_{n}\left|e_{u}^{n}\right|_{1}^{2}+C_{\gamma} \sum_{n=1}^{k} \int_{t_{n-1}}^{t_{n}}\left[1+\left|u_{\varepsilon}\right|_{1, \infty}^{2}\right] d t\left\|\mathcal{G} e_{v}^{n}\right\|_{1}^{2}+C h^{2} .
\end{aligned}
$$

The error $\left(\mathcal{V}_{3}^{k}\right)_{5}$ is due solely to the perturbation of domain between $\Omega$ and $\Omega^{h}$ and is bounded, on noting (3.9) and (3.14a), by

$$
\left(\mathcal{V}_{3}^{k}\right)_{5} \leq C \sum_{n=1}^{k} \tau_{n}\left[h^{2}\left\|\left[D\left(I^{h}\left[b\left(U_{\varepsilon}^{n}\right)\right]\right)\right]^{\frac{1}{2}} \nabla W_{\varepsilon}^{n}\right\|_{0, \Omega^{h}}^{2}+\left\|\mathcal{G} e_{v}^{n}\right\|_{1}^{2}\right] .
$$

Similarly to (3.76) and (3.55), we have, on noting (3.17) and (1.2), for any $x \in \kappa$ that

$$
\begin{aligned}
\| D\left(I^{h}\left[b\left(U_{\varepsilon}^{n}\right)\right]\right)-I^{h}[ & \left.b\left(U_{\varepsilon}^{n}\right)\right] \mathcal{I} \|_{0, \infty, \kappa} \leq C h_{\kappa}\left|I^{h}\left[b\left(U_{\varepsilon}^{n}\right)\right]\right|_{1, \infty, \kappa} \\
& \leq C h_{\kappa}\left|b\left(U_{\varepsilon}^{n}\right)\right|_{1, \infty, \kappa} \leq C h_{\kappa}\left|b^{\prime}\left(U_{\varepsilon}^{n}\right)\right|_{0, \infty, \kappa}\left|U_{\varepsilon}^{n}\right|_{1, \infty, \kappa} \\
& \leq C h_{\kappa} \sqrt{\left[b\left(U_{\varepsilon}^{n}\right)\right](x)}\left|U_{\varepsilon}^{n}\right|_{1, \infty, \kappa}+C h_{\kappa}^{2}\left|U_{\varepsilon}^{n}\right|_{1, \infty, \kappa}^{2} .
\end{aligned}
$$

Combining (3.80) and (3.81), and recalling (3.5a), (3.28), (3.59), (3.23a) and (3.23b), yields that

$$
\left(\mathcal{V}_{3}^{k}\right)_{5} \leq C\left[h^{2}+\sum_{n=1}^{k} \tau_{n}\left\|\mathcal{G} e_{v}^{n}\right\|_{1}^{2}\right] .
$$

It follows, on noting the monotonicity of $\psi$, that

$$
\begin{aligned}
\left(\mathcal{V}_{3}^{k}\right)_{6} & \leq \gamma \sum_{n=1}^{k} \int_{t_{n-1}}^{t_{n}}\left\|\left[b\left(u_{\varepsilon}\right)\right]^{\frac{1}{2}}\left[\psi_{\varepsilon}\left(v_{\varepsilon}\right)-\psi_{\varepsilon}\left(V_{\varepsilon}^{n}\right)\right]\right\|_{0}^{2} d t+C_{\gamma} \sum_{n=1}^{k} \tau_{n}\left\|\mathcal{G} e_{v}^{n}\right\|_{0}^{2} \\
& \leq \gamma C \sum_{n=1}^{k} \int_{t_{n-1}}^{t_{n}}\left(b\left(u_{\varepsilon}\right)\left[\psi_{\varepsilon}\left(v_{\varepsilon}\right)-\psi_{\varepsilon}\left(V_{\varepsilon}^{n}\right)\right], v_{\varepsilon}-V_{\varepsilon}^{n}\right) d t+C_{\gamma} \sum_{n=1}^{k} \tau_{n}\left\|\mathcal{G} e_{v}^{n}\right\|_{0}^{2} .
\end{aligned}
$$

On performing integration by parts in space on the $\left(\mathcal{V}_{3}^{k}\right)_{7}$ term, we have, on noting (2.18a), (2.18b), (2.18d), (3.14b) and (3.28), that

$$
\begin{aligned}
\left|\left(\mathcal{V}_{3}^{k}\right)_{7}\right|+\left|\left(\mathcal{V}_{3}^{k}\right)_{8}\right| & \leq \sup _{n=1 \rightarrow k}\left\|\left(\mathcal{G}-\mathcal{G}^{h}\right) e_{v}^{n}\right\|_{0}\left[1+\int_{0}^{t_{k}}\left\|\nabla \cdot\left(w_{\varepsilon} \nabla\left[b\left(u_{\varepsilon}\right)\right]\right)\right\|_{0}\right] \\
& \leq C h^{2} \int_{0}^{t_{k}}\left[1+\left\|\nabla w_{\varepsilon}\right\|_{0}^{2}+\left\|\nabla u_{\varepsilon}\right\|_{0, \infty}^{2}+\left\|\Delta u_{\varepsilon}\right\|_{0}\right] d t \\
& \leq C h^{2} .
\end{aligned}
$$


We now combine the above bounds $\sum_{n=1}^{k} \mathcal{V}_{3}^{n}=\sum_{i=1}^{8}\left(\mathcal{V}_{3}^{k}\right)_{i}$ with (3.41)-(3.45) and insert them into (3.40). If we choose $\gamma$ sufficiently small, then we obtain for any integer $k \in[1, N]$ and any $\tilde{\gamma}>0$, on noting (2.18c), that

$$
\begin{aligned}
&\left\|\mathcal{G} e_{v}^{k}\right\|_{1}^{2}+\left.\sum_{n=1}^{k} \int_{t_{n-1}}^{t_{n}}\left(b\left(u_{\varepsilon}\right)\left[\psi_{\varepsilon}\left(v_{\varepsilon}\right)\right)-\psi_{\varepsilon}\left(V_{\varepsilon}^{n}\right)\right], v_{\varepsilon}-V_{\varepsilon}^{n}\right) d t \\
& \leq \tilde{\gamma} \sum_{n=1}^{k} \tau_{n}\left|e_{u}^{n}\right|_{1}^{2}+C\left(\tau+\left[\tau_{\min }\right]^{-1} h^{2}+\left[\psi_{\varepsilon}^{\prime}(0)\right]^{-1} h^{2}+\left\|\mathcal{G} e_{v}^{0}\right\|_{1}^{2}\right) \\
& \quad+C_{\tilde{\gamma}} \sum_{n=1}^{k}\left(\int_{t_{n-1}}^{t_{n}}\left(1+\left|u_{\varepsilon}\right|_{1, \infty}^{2}\right) d t\right)\left(\left\|e_{u}^{n}\right\|_{0}^{2}+\left\|\mathcal{G} e_{v}^{n}\right\|_{1}^{2}\right) .
\end{aligned}
$$

If we now combine (3.39) and (3.85) and choose $\tilde{\gamma}$ small enough, we finally have for any integer $k \in[1, N]$ that

$$
\begin{aligned}
& \left\|e_{u}^{k}\right\|_{0}^{2}+\left\|\mathcal{G} e_{v}^{k}\right\|_{1}^{2}+\sum_{n=1}^{k} \tau_{n}\left|e_{u}^{n}\right|_{1}^{2} \\
& \left.+\sum_{n=1}^{k} \int_{t_{n-1}}^{t_{n}}\left(b\left(u_{\varepsilon}\right)\left[\psi_{\varepsilon}\left(v_{\varepsilon}\right)\right)-\psi_{\varepsilon}\left(V_{\varepsilon}^{n}\right)\right], v_{\varepsilon}-V_{\varepsilon}^{n}\right) d t \\
& \leq C \\
& \quad\left(\tau+\left[\tau_{\min }\right]^{-1} h^{2}+\left[\psi_{\varepsilon}^{\prime}(0)\right]^{-1} h^{2}+\left\|e_{u}^{0}\right\|_{0}^{2}+\left\|\mathcal{G} e_{v}^{0}\right\|_{1}^{2}\right) \\
& \quad+C \sum_{n=1}^{k}\left(\int_{t_{n-1}}^{t_{n}}\left(1+\left|u_{\varepsilon}\right|_{1, \infty}^{2}\right) d t\right)\left(\left\|e_{u}^{n}\right\|_{0}^{2}+\left\|\mathcal{G} e_{v}^{n}\right\|_{1}^{2}\right) .
\end{aligned}
$$

In order to apply the discrete Gronwall inequality (3.27), we require $\tau$ to be sufficiently small. In view of (2.18a) and since $p^{*}>2$, we have that for any integer $k \in[1, N]$

$$
\begin{aligned}
C \int_{t_{k-1}}^{t_{k}}\left(1+\left|u_{\varepsilon}\right|_{1, \infty}^{2}\right) d t & \leq C\left[\tau_{k}+\left(\int_{t_{k-1}}^{t_{k}}\left|u_{\varepsilon}\right|_{1, \infty}^{p^{*}} d t\right)^{\frac{2}{p^{*}}} \tau_{k}^{1-\frac{2}{p^{*}}}\right] \\
& \leq C \tau^{1-\frac{2}{p^{*}}} \leq \frac{1}{2}
\end{aligned}
$$

provided that $\tau \leq \tau_{0}$. Hence we may apply (3.27) with $\left(r^{k}\right)^{2}=\left\|e_{u}^{k}\right\|_{0}^{2}+\left\|\mathcal{G} e_{v}^{k}\right\|_{1}^{2}$, $\left(s^{k}\right)^{2}=\sum_{n=1}^{k}\left[\tau_{n}\left|e_{u}^{n}\right|_{1}^{2}+\int_{t_{n-1}}^{t_{n}}\left(b\left(u_{\varepsilon}\right)\left[\psi_{\varepsilon}\left(v_{\varepsilon}\right)-\psi_{\varepsilon}\left(V_{\varepsilon}^{n}\right)\right], v_{\varepsilon}-V_{\varepsilon}^{n}\right) d t\right], \quad\left(\xi^{n}\right)^{2}=$ $C \int_{t_{n-1}}^{t_{n}}\left(1+\left|u_{\varepsilon}\right|_{1, \infty}^{2}\right) d t$ and $\left(q^{n}\right)^{2}=C \tau_{n}\left(\tau+\left(\left[\tau_{\min }\right]^{-1}+\left[\psi_{\varepsilon}^{\prime}(0)\right]^{-1}\right) h^{2}\right)$; and the desired result (3.29) follows, on noting (2.18a), (3.11) and (1.5).

Remark 3.3. The error bound (3.29) differs from the one obtained in Theorem 3 in Nochetto and Verdi ${ }^{8}$ in that the term $\left[\tau_{\min }\right]^{-1} h^{2}$ in (3.29) is replaced by the term $\left(\left[\psi_{\varepsilon}^{\prime}(0)\right]^{2} \tau_{\min }\right)^{-1} h^{4}$ in Nochetto and Verdi. ${ }^{8}$ This is because the term $\mathcal{V}_{2}^{n}$ is bounded in Nochetto and Verdi ${ }^{8}$ with the help of a bound on $\psi_{\varepsilon}^{\prime}(0) \sum_{n=1}^{N}\left\|V_{\varepsilon}^{n}-V_{\varepsilon}^{n-1}\right\|_{0}^{2}$. Due to the presence of the (possibly degenerate) function $b(u)$, such an estimate is 
not available in our case. As regards the optimality of the error bound (3.29), that is an open question.

We are now in a position to bound the error between the solution $(u, v)$ of $(\mathrm{P})$ and its discrete approximation $\left(U_{\varepsilon}, V_{\varepsilon}\right)$, where we define $\left(U_{\varepsilon}, V_{\varepsilon}\right)(t)=\left(U_{\varepsilon}^{n}, V_{\varepsilon}^{n}\right)$ for $t \in\left(t_{n-1}, t_{n}\right], n=1 \rightarrow N$.

Theorem 3.3. Let the assumptions of Theorem 3.2 hold. In addition, we assume that $\tau \leq C \tau_{\text {min }}$. Let $h=\tau=\varepsilon q_{\varepsilon}^{2}$ (where $q_{\varepsilon}$ is as defined for (2.2)) if $\psi$ is degenerate, and $h=\tau$ otherwise. Then we have that

$$
\sup _{t \in(0, T)}\left\|\left(u-U_{\varepsilon}\right)(\cdot, t)\right\|_{0}^{2}+\int_{0}^{T}\left|u-U_{\varepsilon}\right|_{1}^{2} d t+\sup _{t \in(0, T)}\left\|\left(v-V_{\varepsilon}\right)(\cdot, t)\right\|_{\left(H^{1}\right)^{\prime}}^{2} \leq C h .
$$

Furthermore, if $K$ is a compact subset of $\left\{(x, t) \in \Omega_{T}:[b(u)](x, t)>0\right\}$, then there exists $C_{K}>0$ such that

$$
\left\|v-V_{\varepsilon}\right\|_{0, K} \leq \begin{cases}C_{K} q_{\varepsilon} & \text { if } \psi \text { is degenerate, } \\ C_{K} h^{\frac{1}{2}} & \text { if } \psi \text { is non-degenerate. }\end{cases}
$$

Proof. Note first that $q_{\varepsilon}=0$ if $\psi$ is non-degenerate. The first bound follows immediately from combining (2.32) and (3.29), and recalling (3.25), (2.18a) and (2.18c).

Next, since $u \in C\left(\overline{\Omega_{T}}\right)$, there exists $\delta_{K}>0$ such that $[b(u)](x, t) \geq \delta_{K}$ for all $(x, t) \in K$. Suppose first that $\psi$ is degenerate. We infer from (2.32) that

$$
\varepsilon \delta_{K} \int_{K}\left(v-v_{\varepsilon}\right)^{2} \leq \delta_{K} \int_{K}\left[\psi_{\varepsilon}(v)-\psi_{\varepsilon}\left(v_{\varepsilon}\right)\right]\left(v-v_{\varepsilon}\right) \leq C \varepsilon q_{\varepsilon}^{2} .
$$

On the other hand we have from (2.18d), (2.19), (3.28), (1.2) and the monotonicity of $\psi_{\varepsilon}$ that

$$
\begin{aligned}
&\left(b(u)\left[\psi_{\varepsilon}\left(v_{\varepsilon}\right)-\psi_{\varepsilon}\left(V_{\varepsilon}\right)\right], v_{\varepsilon}-V_{\varepsilon}\right) \\
&=\left(\left[b\left(u_{\varepsilon}\right)+\left(b(u)-b\left(u_{\varepsilon}\right)\right)\right]\left[\psi_{\varepsilon}\left(v_{\varepsilon}\right)-\psi_{\varepsilon}\left(V_{\varepsilon}\right)\right], v_{\varepsilon}-V_{\varepsilon}\right) \\
& \leq\left(b\left(u_{\varepsilon}\right)\left[\psi_{\varepsilon}\left(v_{\varepsilon}\right)-\psi_{\varepsilon}\left(V_{\varepsilon}\right)\right], v_{\varepsilon}-V_{\varepsilon}\right) \\
& \quad+\left(\left|b^{\prime}(u)\right|\left|u-u_{\varepsilon}\right|+C\left|u-u_{\varepsilon}\right|^{2},\left|\psi_{\varepsilon}\left(v_{\varepsilon}\right)-\psi_{\varepsilon}\left(V_{\varepsilon}\right)\right|\right) \\
& \leq\left(b\left(u_{\varepsilon}\right)\left[\psi_{\varepsilon}\left(v_{\varepsilon}\right)-\psi_{\varepsilon}\left(V_{\varepsilon}\right)\right], v_{\varepsilon}-V_{\varepsilon}\right) \\
& \quad+\gamma\left(b(u)\left[\psi_{\varepsilon}\left(v_{\varepsilon}\right)-\psi_{\varepsilon}\left(V_{\varepsilon}\right)\right], v_{\varepsilon}-V_{\varepsilon}\right)+C_{\gamma}\left\|u-u_{\varepsilon}\right\|_{0}^{2} .
\end{aligned}
$$

If we choose $\gamma$ sufficiently small and use again (2.32) and (3.29), we finally obtain

$$
\varepsilon \delta_{K} \int_{K}\left(v_{\varepsilon}-V_{\varepsilon}\right)^{2} \leq \int_{0}^{T}\left(b(u)\left[\psi_{\varepsilon}\left(v_{\varepsilon}\right)-\psi_{\varepsilon}\left(V_{\varepsilon}\right)\right], v_{\varepsilon}-V_{\varepsilon}\right) d t \leq C \varepsilon q_{\varepsilon}^{2} .
$$

Combining (3.88) and (3.90) yields the desired result (3.87b) in the degenerate case. If $\psi$ is non-degenerate then (3.87b) follows immediately from (3.89) and (3.29). 
Remark 3.4. If $\psi(s)=s^{p}$ with $p>1$, then $q_{\varepsilon}=\left(\frac{\varepsilon}{p}\right)^{\frac{1}{p-1}}$. In addition, it is easily deduced that

$$
\left[\psi_{\varepsilon}(s)-\psi_{\varepsilon}(r)\right](s-r) \geq(s-r)^{p+1} \quad \forall r, s \in \mathbb{R}_{\geq 0} .
$$

Hence one can use this bound in (3.88) and (3.90) to obtain, in place of (3.87b), for $\psi(s)=s^{p}$ with $p \geq 1$ that

$$
\left\|v-V_{\varepsilon}\right\|_{0, p+1, K} \leq C_{K} h^{\frac{1}{p+1}} .
$$

\section{References}

1. Barrett, J.W. \& NÜrnberg, R. Finite element approximation of a nonlinear degenerate parabolic system describing bacterial pattern formation. Interfaces and Free Boundaries 4, (2002) 277-307.

2. Barrett, J.W. \& Nürnberg, R. Finite element approximation of a Stefan problem with degenerate joule heating. M2AN Math. Model. Numer. Anal. 38, (2004) 633-652.

3. Elliott, C.M. Error analysis of the enthalpy method for the Stefan problem. IMA J. Numer. Anal. 7, (1987) 61-71.

4. Golding, I., Kozlovsky, Y., Cohen, I. \& Ben-Jacob, E. Studies of bacterial branching growth using reaction-diffusion models for colonial development. Physica A 260, (1998) 510-554.

5. Kawasaki, K., Mochizuki, A., Matsushita, M., Umeda, T. \& Shigesada, N. Modelling spatio-temporal patterns generated by bacillus subtilis. J. Theor. Biol. 188, (1997) 177-185.

6. Ladyzhenskaya, O.A., Solonnikov, V.A. \& Uraltseva, N.N. Linear and Quasilinear Equations of Parabolic Type, Transl. Math. Monographs, vol. 23 (Providence, RI: AMS, 1968).

7. Mimura, M., Sakaguchi, H. \& Matsushita, M. Reaction-diffusion modelling of bacterial colony patterns. Physica A 282, (2000) 283-303.

8. Nochetto, R.H. \& Verdi, C. Approximation of degenerate parabolic problems using numerical integration. SIAM J. Numer. Anal. 25, (1988) 784-814.

9. Ortega, J.M. \& Rheinboldt, W.C. Iterative Solution of Nonlinear Equations in Several Variables, (New York: Academic Press, 1970).

10. Simon, J. Compact sets in $L^{p}(0, T ; B)$. Ann. Mat. Pura Appl. 146, (1987) 65-96.

11. Solonnikov, V.A. A priori estimates for second order parabolic equations. Transl. AMS 65, (1967) 51-137.

12. Ziemer, W.P. Weakly Differentiable Functions, (New York: Springer-Verlag, 1989). 